\title{
FULL CONTINUOUS EMBEDDINGS OF TOPOSES
}

\author{
BY \\ M. MAKKAI ${ }^{1}$
}

\begin{abstract}
Some years ago, G. Reyes and the author described a theory relating first order logic and (Grothendieck) toposes. This theory, together with standard results and methods of model theory, is applied in the present paper to give positive and negative results concerning the existence of certain kinds of embeddings of toposes. A new class, that of prime-generated toposes is introduced; this class includes M. Barr's regular epimorphism sheaf toposes as well as the so-called atomic toposes introduced by M. Barr and R. Diaconescu. The main result of the paper says that every coherent prime-generated topos can be fully and continuously embedded in a functor category. This result generalizes M. Barr's full exact embedding theorem. The proof, even when specialized to Barr's context, is essentially different from Barr's original proof. A simplified and sharpened form of Barr's proof of his theorem is also described. An example due to J. Malitz is adapted to show that a connected atomic topos may have no points at all; this shows that some coherence assumption in our main result is essential.
\end{abstract}

Introduction. This paper has three sources. One is Michael Barr's full exact embedding theorem [2] for exact categories, generalizing the Lubkin-FreydMitchell embedding theorems for Abelian categories. Another is the theory of (Grothendieck) toposes, especially coherent toposes (see SGA4 [1]) and the third is model theory (see CK [4]). We have found a particular kind of topos, the so-called prime-generated ones that comprise both the regular epimorphism sheaf toposes related to Barr's regular categories and atomic toposes introduced in Barr and Diaconescu [3] (also considered by A. Joyal). We prove a full embedding theorem for coherent prime-generated toposes that generalizes Barr's theorem and specializes in a new result for coherent atomic toposes. Our chief tools are model theoretical; we use the mechanisms introduced in [10] relating categories and logic.

Specifically, we call an object $A$ of a topos $\mathcal{E}$ a prime object if it cannot be written as a supremum of proper subobjects. $\mathcal{E}$ is a prime-generated topos, if the prime objects in $\mathscr{E}$ form a collection of generators for $\mathscr{E}$. For toposes $\mathscr{E}, \mathcal{E}^{\prime}$ a functor $\mathcal{E} \rightarrow \mathcal{E}^{\prime}$ is continuous if it is left exact and has a right adjoint, i.e. if it is the inverse-image functor of a geometric morphism $\mathcal{E}^{\prime} \rightarrow \mathcal{E}$. Our main results are that every prime-generated coherent topos admits a full continuous embedding into a functor category $(K$, SET) with a small category $K$ (Corollary 2.7$)$ and that, in fact,

Received by the editors June 4, 1979.

1980 Mathematics Subject Classification. Primary 03G30, 18B15; Secondary 03C50.

Key words and phrases. Grothendieck topos, geometric morphism, functor category, full embedding, prime-generated topos, atomic topos, coherent topos, special model, exact category.

${ }^{1}$ The author's research was supported by a grant of the Natural Sciences and Engineering Research Council of Canada. 
the canonically defined evaluation functor ev: $\mathcal{E} \rightarrow(\operatorname{Con}(\mathcal{E}$, SET $)$, SET $)$ with the category $\operatorname{Con}(\mathcal{E}$, SET) of all continuous functors $\mathcal{E} \rightarrow$ SET is continuous, faithful and full (Theorem 2.9).

Given $\mathcal{R}$, a finitely complete regular category in the sense of Barr [2], the topos $\tilde{\mathcal{R}}$ of regular epimorphism sheaves over $\Re$ is easily seen to be prime-generated and coherent; the canonical full exact embedding $\mathcal{R} \rightarrow \tilde{\mathcal{R}}$ composed with the one given by the first of the above results shows that every finitely complete regular category has a full exact embedding into a functor category, which is Barr's main result in [2].

A special kind of prime-generated topos (different from the regular epimorphism sheaf toposes) are atomic toposes: ones that are generated by atoms, i.e., objects $A$ such that $A$ does not have any subobject besides $A$ itself and 0 . Atomic toposes are introduced in [3] where they are shown to be the answer to a certain characterization problem involving the global section functor.

At the end of $\$ 3$ and in $\$ 5$, we point out that atomic toposes come up naturally in model theory; e.g., the classifying toposes of $\boldsymbol{\kappa}_{0}$-categorical (countable) theories are coherent atomic toposes. The special cases for atomic toposes of our main results in $\$ 2$ have simpler proofs described in $\$ 3$; at the same time, sharper results are obtained for them. We obtain the representation theorem that every connected atomic topos that is either coherent or separable is equivalent to the category of discrete $G$-sets of a topological group $G$ (Corollary 3.2 and Theorem 3.8; a topos is connected if its terminal object is an atom). This compares with an earlier result of Joyal that is weaker but more general than ours (see below). The fact that not every connected atomic topos can have such a representation is dramatized by the fact that there are connected atomic toposes without points (without continuous functors to SET) (it was Gonzalo Reyes who first asked the question if this was the case); in $\$ 5$ we give a description of an example originating in Malitz [14] and Gregory [5]. In $\S 3$, we briefly mention the connection of results related to the above representation theorem with the question if "the category of models determines the theory", a problem being currently investigated.

In §4, we give another proof of Barr's full exact embedding theorem (our third one, see also [11]). We have arrived at this proof by trying to understand Barr's original proof; despite the differences in formulation, this proof can safely be called "Barr's proof". We also believe that our formulation is easier to follow (especially for model theorists).

There are coherent toposes that admit full continuous embeddings into functor categories but which are not prime-generated. In fact, our methods in $\$ 2$ are applicable to a larger class of coherent toposes. The description of this class however involves the introduction of the prime completion of a coherent topos which, if properly done, would take up much space. We have decided to describe the special case of prime-generated toposes here with a minimum of formal details and defer the general treatment of the prime completion to another paper [13].

1. Preliminaries. For general logical terminology, we refer to CK [4] and MR [10]. All the necessary material concerning categories and categorical logic can be 
found in MR. Here we try to recall enough of the basic terminology and facts to make the paper reasonably self-contained. Fundamental topos-theoretical concepts however will be taken for granted; MR is a sufficient reference.

Model-theoretical concepts will be considered in the context of many-sorted languages; also, the 'partial domains' of the structures, i.e., the interpretations of the sorts, are allowed to be empty. Both the notions and the theorems we need from CK have completely straightforward extensions to the many-sorted situation with possibly empty domains.

Most frequently, the language $L$ considered will be the underlying graph of a category $\mathcal{C}$; in other words, the sorts of the language are the objects of $\mathcal{C}$, and the only other data are the unary operation symbols that are the morphisms of $\mathcal{C}$. Of course, it is a basic point that functors $e \rightarrow$ SET are $L$-structures in this case; actually, $L$-structures are precisely what could be called functors from $L(=$ the underlying graph of $\mathcal{C}$ ) to SET (where a functor from a graph to, say, SET has the same definition as that of a functor from a category to SET, except that reference to composition and identity is deleted). Adhering to the functorial notation, for an arbitrary language $L$, we denote by $M(X)$ the interpretation of the sort $X$ ('partial domain') in $M$, etc. $|L|$ denotes the set of sorts of $L$. Unless otherwise mentioned, all structures below are $L$-structures for a fixed $L$.

One fact that follows from MR is that there is no loss of generality from the point of view of logic when one restricts attention to languages that are underlying graphs of categories.

Except in $\$ 5$, "formula" and other like notions refer to $L_{\omega \omega}$, the full finitary first order logic over $L$, unless otherwise mentioned. A homomorphism $h: M \rightarrow N$ of $L$-structures (i.e., a natural transformation $h: M \rightarrow N$, in case $M, N$ are functors $\mathcal{C} \rightarrow \mathrm{SET})$ is an elementary embedding if it preserves all formulas: whenever $\varphi\left(x_{1}, \ldots, x_{n}\right)$ is a formula with the indicated free variables, $x_{i}$ of sort $X_{i}, a_{i} \in$ $M\left(X_{i}\right), \vec{a}=\left\langle a_{1}, \ldots, a_{n}\right\rangle, h \vec{a}=\left\langle h_{X_{1}}\left(a_{1}\right), \ldots, h_{X_{n}}\left(a_{n}\right)\right\rangle$, then $M \vDash \varphi[\vec{a}](\vec{a}$ satisfies $\varphi$ in $M$ ) implies that $N \vDash \varphi[h \vec{a}]$. Note that an elementary embedding $h: M \rightarrow N$ is injective, i.e., for every $X \in|L|$, the function $h_{X}: M(X) \rightarrow N(X)$ is one-to-one.

A primitive formula is one built up from atomic formulas using finite conjunction and existential quantification (in any order). Note that any homomorphism preserves every primitive formula.

The underlying set of a structure $M$ is $|M|=\amalg_{X \in|L|} M(X)$, the disjoint sum of the partial domains of $M$. Without loss of generality, one can assume that the sets $M(X)(X \in|L|)$ are already pairwise disjoint (every structure is isomorphic to one such), and then $|M|$ becomes $\cup_{X \in|L|} M(X)$, the set of elements of $M$. The cardinality of $M, \operatorname{card} M$, is the cardinality of the set $|M|$.

In CK, p. 217, the notion of a special structure is introduced, for the 'one-sorted' case. Trivial and minor modifications are all that are needed to formulate the notion for the general case, as well as to prove the basic results. Instead of describing the notion in detail, we summarize the facts we need in the following theorem and 1.2 below. 
THEOREM 1.1. Let a language $L$ be given. We have a class of cardinals, called special cardinals, and for each special cardinal $\lambda$, a class of L-structures called $\lambda$-special structures with the following properties:

(i) There are arbitrarily large special cardinals.

(ii) Every $\lambda$-special structure is of cardinality $\leqslant \lambda$.

(iii) Every structure of cardinality $\leqslant \lambda$ has an elementary embedding into a $\lambda$-special structure.

(iv) Whenever $\vec{a}$ and $\vec{b}$ are finite tuples of elements of $M, a \lambda$-special structure for some $\lambda$, such that $M \vDash \varphi[\vec{a}]$ implies $M \vDash \varphi[\vec{b}]$ for all formulas $\varphi(\vec{x})$, then there is an automorphism $h$ of $M$ such that $h(\vec{a})=\vec{b}$.

(v) Whenever card $M \leqslant \lambda$ and $N$ is $\lambda$-special, moreover, $\vec{a}$ is a tuple from $M, \vec{b}$ from $N$ such that for any primitive formula $\varphi(\vec{x}), M \vDash \varphi[\vec{a}]$ implies $N \vDash \varphi[\vec{b}]$, then there is a homomorphism $h: M \rightarrow N$ such that $h(\vec{a})=\vec{b}$.

A few remarks are in order. For the reader who wants to make a comparison with CK, we note that a $\lambda$-special structure is one that is of power $\leqslant \lambda$ and that is the union (directed limit) of an elementary chain of structures $M_{\kappa}$ indexed by cardinals $\kappa<\lambda$ such that $M_{\kappa}$ is $\kappa^{+}$-saturated, for each $\kappa<\lambda$; note the slight change with respect to $\mathrm{CK}$ resulting in the introduction of the prefix $\lambda$-. Part (iii) in 1.1 is 5.1.8 in CK; (iv) is an equivalent formulation of 5.1.17 (to realize that 5.1.17 does give (iv), note the simple fact that the expansion of a special structure by finitely many elements is special). Finally, the proof of (v) is quite analogous to that of (iii); also, similar arguments are found in CK in $\$ 5.2$ leading to Proposition 5.2.11.

Next we turn to category-theoretic notions and their relations to logic. Every topos here is a Grothendieck topos (in distinction to elementary toposes). Toposes, coherent toposes, etc. are all introduced in SGA4; MR also contains all we need.

For toposes $\mathcal{E}, \mathcal{E}^{\prime}$, the continuous functors $\mathcal{E} \rightarrow \mathcal{E}^{\prime}$ are those that are left exact and preserve all coverings of the canonical topology on $\mathcal{E}$; they are called $\infty$-logical in MR and they are also identical to the inverse-image functors of geometric morphisms $\mathcal{E}^{\prime} \rightarrow \mathcal{E}$ (compare [6]). The category of continuous functors $\mathcal{E} \rightarrow \mathcal{E}^{\prime}$ is denoted by $\operatorname{Con}\left(\mathcal{E}, \mathcal{E}^{\prime}\right)$, with arbitrary natural transformations as morphisms. Sometimes continuous functors $\mathcal{E} \rightarrow$ SET are called models of $\mathcal{E}$.

Until further notice, let $\mathcal{E}$ be a fixed coherent topos, $\mathscr{P}=\operatorname{Coh}(\mathcal{E})$ (a small category equivalent to) the category of coherent objects of $\mathcal{E}$ (a full subcategory of $\mathcal{E}$ ). The continuous functors $\mathcal{E} \rightarrow \mathcal{E}^{\prime}$ (for any $\mathcal{E}^{\prime}$ ) are essentially in a one-one correspondence with the functors $\mathscr{P} \rightarrow \mathcal{E}^{\prime}$ that are called 'logical' in MR. Unfortunately, the terminology is in conflict with a widespread use of the word 'logical' for a different meaning (compare [6]); instead, we will talk about elementary functors. The elementary functors are those that preserve finite (left) limits (they are left exact), images (extremal epis, also called surjective morphisms in MR), and finite sups (of subobjects). The category of elementary functors $\mathcal{P} \rightarrow \mathcal{E}^{\prime}$ is denoted by $\operatorname{El}\left(\mathcal{P}, \mathcal{E}^{\prime}\right)$. The precise statement of what we said above is that the 'restriction' functor

$$
\rho: \operatorname{Con}\left(\mathcal{E}, \mathcal{E}^{\prime}\right) \rightarrow \operatorname{El}\left(\mathscr{P}, \mathcal{E}^{\prime}\right)
$$


(for which $\rho(F)=F \circ I$, with the inclusion $I: \mathcal{P} \rightarrow \mathcal{E}$ ) is an equivalence of categories.

On the other hand, the elementary functors $\mathscr{P} \rightarrow$ SET (literally) coincide with the models of a certain theory $T_{\mathscr{\Phi}}$, and in fact, the elementary functors $\mathcal{P} \rightarrow \mathcal{E}^{\prime}$ for an arbitrary topos $\mathcal{E}^{\prime}$ coincide with the $\mathcal{E}^{\prime}$-models of $T_{\mathscr{\Phi}}$ (as explained in MR). The language of $T_{\mathscr{P}}$ is the underlying graph of $\mathscr{P}$, and the axioms of $T_{\mathscr{P}}$ all have the form $\forall \vec{x}(\varphi(\vec{x}) \rightarrow \psi(\vec{x}))$ where $\varphi, \psi$ are so-called coherent formulas, i.e. built up from atomic formulas using only finite disjunction, finite conjunction and existential quantification.

We will be interested in special models of $T_{\mathscr{\rho}}$. We call the continuous functor $M$ : $\mathcal{E} \rightarrow$ SET $\lambda$-special if the composite (restriction) $M_{0}: \mathscr{P} \rightarrow$ incl. $\mathscr{E} \rightarrow$ SET is $\lambda$ special.

Occasionally, we will use a special case of the general concept of interpreting formulas in categories as explained in MR. Given a category $\mathcal{C}$, a formula $\varphi$ in the language that is the underlying graph of $\mathcal{C}$, and $\vec{x}$ is a finite sequence of variables including the free variables in $\varphi,[\varphi]_{\vec{x}}$ is the canonical interpretation of $\varphi$ in $\mathcal{C}$ : a certain subobject of $X=X_{1} \times \cdots \times X_{n}$ where $\vec{x}=\left\langle x_{1}, \ldots, x_{n}\right\rangle, x_{i}$ of sort $X_{i}$ $(i=1, \ldots, n)$. The definition of $[\varphi]_{\vec{x}}$ is based on the 'logical operations' in $\mathcal{C}$; e.g. if $\mathcal{C}$ is a pretopos, $[\varphi]_{\vec{x}}$ is defined for all coherent formulas $\varphi$. If $M: \mathcal{C} \rightarrow$ SET is elementary, $\varphi$ is coherent and $M_{\vec{x}}(\varphi)=\{\vec{a} \in M(X): M \vDash \varphi[\vec{a}]\} \subset M(X)$, then $M_{\vec{x}}(\varphi)$ coincides with $M\left([\varphi]_{\vec{x}}\right) \subset M(X)$. If $A \hookrightarrow^{\alpha} Y$ is a subobject of an object $Y$, $\mathbf{A}(y)$ denotes a formula, with a variable $y$ of sort $Y$, such that $[\mathbf{A}(y)]_{y}=A ; \mathbf{A}(y)$ can be taken to be $\exists a(\alpha(a)=y)$.

Regular and exact categories are defined in [2]; we will consider only finitely complete regular categories. We briefly recall some of the notions involved. A category is finitely complete if it has finite left limits. An extremal epi is a morphism that does not factor into two morphisms the second of which is a monomorphism unless the latter is an isomorphism. An extremal epi is stable if all its pullbacks are extremal epis as well. A finitely complete category is regular just in case it has stable images, i.e. the extremal epis are stable and every morphism factors into an extremal epi followed by a mono. We note that, at least in a finitely complete regular category as defined here, extremal epis are the same as regular epis of [2]; this statement is contained in Proposition 3.3.3 in MR. A functor is exact if it is left exact and preserves extremal epis. The category of all exact functors $R \rightarrow \Omega^{\prime}$ (with arbitrary natural transformations) is denoted by $\operatorname{Ex}\left(\Re, \Re^{\prime}\right)$.

The general considerations of $\$ 2.4$ in MR give us, for any finitely complete regular category $\Re$, a theory $T_{\mathscr{R}}^{\text {reg }}$, the internal (regular) theory of $\mathscr{R}$, such that $T_{\mathscr{R}}^{\text {reg }}$ is formulated in the language that is the underlying graph of $R$ and such that the models of $T_{\mathscr{R}}^{\text {reg }}$ are precisely the exact functors $\mathcal{R} \rightarrow$ SET. All axioms of $T_{\mathscr{R}}^{\text {reg }}$ are of the form $\forall \vec{x}(\varphi(\vec{x}) \rightarrow \psi(\vec{x}))$ where $\varphi$ and $\psi$ are primitive formulas (no disjunction is involved); the axioms are the ones that are described in loc.cit. as to correspond to the finite left limit diagrams of $\mathscr{R}$ and to the extremal epis of $\Re$ (item 9 on p. 92). 
Also, if $\varphi$ is any primitive formula in the same language, the canonical interpretation $[\varphi]_{\vec{x}}$ of $\varphi$ exists in $\Re$ and has the expected properties as described above.

A continuous functor $F: \mathcal{E} \rightarrow \mathcal{E}^{\prime}$ between toposes is faithful just in case it is conservative: whenever $A$ is a proper subobject of $X$ in $\mathcal{E}, F(A)$ is a proper subobject of $F(X)$ as well. We want to discuss conservativeness in a special case.

Given an arbitrary subcategory $K$ of $\operatorname{Con}(\mathcal{E}, \mathrm{SET})$ with $\mathcal{E}$ a coherent topos, $\mathscr{P}=\operatorname{Coh}(\mathcal{E})$, one defines, as usual, the evaluation functor $\mathrm{ev}=\mathrm{ev}_{K}: \mathcal{E} \rightarrow$ $(K, \operatorname{SET})$ by $(\operatorname{ev}(E))(M)=M(E)$ for $M \in|K|,(\operatorname{ev}(E))(h)=h_{E}$ for $h: M \rightarrow M^{\prime}$ in $K,(\operatorname{ev}(f))_{M}=M(f)$ for $f: E \rightarrow E^{\prime}$ in $\mathcal{E}$. As it is easy to see (see also $\S 6.3$ in MR), $\mathrm{ev}_{K}$ is continuous. Now, it is conservative just in case the following condition is satisfied: whenever $\left\{A_{i}: i \in I\right\}$ is a family of coherent subobjects of a coherent object $X$, and for every finite $I^{\prime} \subset I, \bigvee_{i \in I^{\prime}} A_{i} \neq X$, then there is $M \in|K|$ such that $M(X)-\cup_{i \in I} M\left(A_{i}\right) \neq \varnothing$ (see $\S 6.2$ in MR). Note in particular that conservativeness has nothing to do with the morphisms in $K$; in case $\mathrm{ev}_{K}$ is conservative, we call $|K|$ a conservative family (of functors). The condition for conservativeness can be paraphrased, in 'logical' terms, as follows: whenever $\left\{\neg \mathbf{A}_{i}(x): i \in I\right\}$ is finitely consistent with the internal theory $T_{\mathscr{P}}$ of $\mathcal{P}$, then there is a model $M$ in $|K|$ that has an element $a \in M(X)$ satisfying each $-\mathbf{A}_{i}(x)(i \in I)$.

Below, we will use a concept 'dual' to conservativeness. For $K$ as above, we call $|K|$ a dually conservative family if the following is satisfied: whenever $\left\{A_{i}: i \in I\right\}$ is a family of coherent subobjects of a coherent object $X$ in $\mathcal{E}, A$ a coherent subobject of $X$, and $\bigcap_{i \in I} M\left(A_{i}\right) \subset M(A)$ for all $M$ in $|K|$, then there is a finite subset $I^{\prime}$ of $I$ such that $\bigwedge_{i \in I^{\prime}} A_{i} \leqslant A$. The 'logical' paraphrase is as follows: whenever $\Theta=\left\{\mathbf{A}_{i}(x): i \in I\right\} \cup\{\neg \mathbf{A}(x)\}$ is finitely consistent with $T_{\mathscr{\rho}}$, then there is $M$ in $|K|$ with an element $a \in M(X)$ satisfying every formula in $\Theta$.

Let $K_{\lambda}$ denote the full subcategory of $\operatorname{Con}(\mathcal{E}, \mathrm{SET})$ consisting of the $\lambda$-special continuous functor $\mathcal{E} \rightarrow$ SET. Then it is easy to see (using the compactness and Löwenheim-Skolem theorems, 1.1(iii), as well as the fact that each special cardinal is larger than the cardinality of the language) that the class $\left|K_{\lambda}\right|$ satisfies both of the above 'logical' paraphrases. In other words, we have

Proposition 1.2. For $\mathcal{E}$ and $K_{\lambda}$ as above, $\left|K_{\lambda}\right|$ is a conservative as well as dually conservative family of continuous functors $\mathcal{E} \rightarrow S E T$.

Finally, one more result we need is Theorem 2.4 .4 (p. 89) in MR.

Proposition 1.3. In every finitely complete category $\mathcal{C}$, every functional relation $R \hookrightarrow X \times Y$ is the graph of a unique morphism. In particular, if $|K|$ is a conservative family of left-exact functors $M: \circlearrowright \rightarrow$ SET such that, for each $M \in|K|, M(R) \hookrightarrow X$ $\times Y$ is the graph of a function $f_{M}$, then there is a morphism $g: X \rightarrow Y$ in $\mathcal{C}$ such that $R$ is the graph of $g$ and as a consequence $M(g)=f_{M}$ for all $M \in|K|$.

\section{Coherent prime-generated toposes.}

Definition 2.1. An object $P$ of a site $C$ is prime if any covering family $\left\{A_{i} \rightarrow P\right.$ : $i \in I\}$ contains a morphism $A_{i} \rightarrow P$ which, by itself, is a covering. A prime object in a topos $\mathcal{E}$ is one that is prime with respect to the site $\mathcal{E}$ with the canonical 
topology; equivalently, $P \in|\mathscr{E}|$ is prime iff $P$ is not the union of the family of its proper subobjects. A site is prime-generated if every object has a covering by morphisms whose domains are prime objects. A topos is prime-generated if it is prime-generated as a site with the canonical topology; in other words, if the family of prime objects is a family of generators.

All categories, in particular, the underlying categories of all sites, are henceforth assumed to be finitely complete.

LEMMA 2.2. In a site, if $\{A \rightarrow B\}$ is a covering (consisting of a single morphism) and $A$ is a prime object, then $B$ is prime as well. In particular, any quotient of a prime object in a topos is prime.

Proof. Suppose $\{A \rightarrow B\},\left\{B_{i} \rightarrow B: i \in I\right\}$ are coverings. Then $\left\{A \times{ }_{B} B_{i} \rightarrow A\right.$ : $i \in I\}$ is a covering; hence for some $i, A \times{ }_{B} B_{i} \rightarrow A$ is a covering. It follows that the composite $f: A \times{ }_{B} B_{i} \rightarrow A \rightarrow B$ is a covering; since $f$ factors through $B_{i} \rightarrow B$, $\left\{B_{i} \rightarrow B\right\}$ is a covering itself.

LEMMA 2.3. Let $\mathcal{E}$ be a coherent prime-generated topos. Then the family of coherent prime objects is a family of generators for $\mathcal{E}$.

Proof. Since the coherent objects form a family of generators for $\mathcal{E}$, it suffices to show that every coherent object in $\mathcal{E}$ is the union of its coherent prime subobjects (i.e., subobjects whose domains are coherent and prime). Since the prime objects generate, and the image of a prime object is prime (by 2.2), every object is the union of its prime subobjects. If $X$ is a coherent object, then every subobject $A$ of $X$ is a union of coherent subobjects (see 6.1.3 in MR); hence if $A$ is in particular prime, then $A$ itself must be coherent; this shows what we wanted.

LEMMA 2.4. Let $F: \mathcal{E} \rightarrow \mathcal{E}^{\prime}$ be a continuous conservative functor between toposes; let $\mathcal{S}$ be a family of generators for $\mathcal{E}$. Suppose that $F$ is full on each hom-set $\operatorname{Hom}_{\mathfrak{E}}(S, E)$ with $S \in \mathcal{S}, E \in|\mathcal{E}|$; in other words, for every morphism g: FS $\rightarrow F E$ there is $f: S \rightarrow E$ such that $g=F(f)$. Then $F$ is full.

Proof. Let $E_{1}, E_{2}$ be objects in $\mathcal{E}, F E_{1} \rightarrow^{f} F E_{2}$ a morphism in $\mathcal{E}^{\prime}$. Let $\left\{S_{i} \rightarrow{ }^{\delta_{i}} E_{1}: i \in I\right\}$ be a (canonical) covering of $E_{1}$ in $\mathcal{E}$ by objects $S_{i}$ in $\mathcal{S}$. Consider the composites $F S_{i} \rightarrow^{F g_{1}} F E_{1} \rightarrow^{f} F E_{2}$; since $F$ is assumed to be full on $\operatorname{Hom}_{\mathscr{E}}\left(S_{i}, E_{2}\right)$, there is $f_{i}: S_{i} \rightarrow E_{2}$ in $\mathcal{E}$ such that $F f_{i}=f \circ F g_{i}(i \in I)$. The family $\left\{f_{i}\right\}_{i \in I}$ is a family of morphisms from the covering $\left(S_{i} \rightarrow^{\boldsymbol{s}_{i}} E_{1}\right)_{i \in I}$ to $E_{2}$; we claim that it is a compatible family, i.e. in

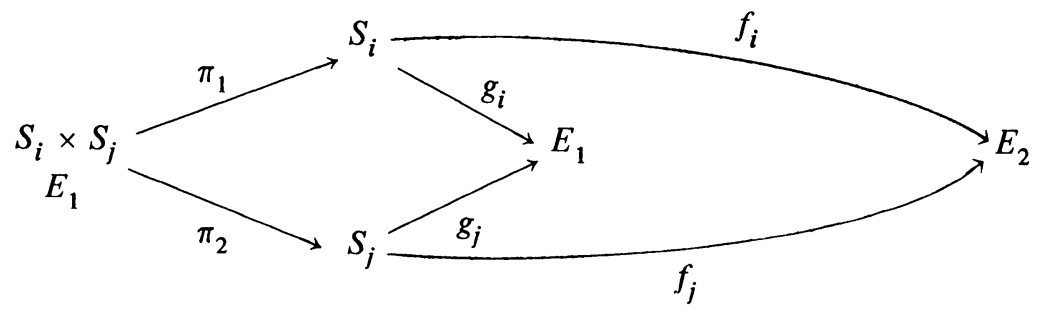


we have $f_{i} \circ \pi_{1}=f_{j} \circ \pi_{2}$. This certainly becomes true when the function $F$ is applied: $\quad F\left(f_{i} \circ \pi_{1}\right)=F\left(f_{i}\right) \circ F \pi_{1}=f \circ F\left(g_{i} \circ \pi_{1}\right)=f \circ F\left(g_{j} \circ \pi_{2}\right)=F\left(f_{j} \circ \pi_{2}\right)$. Since $F$ is conservative, $F$ is faithful; thus $f_{i} \circ \pi_{1}=f_{j} \circ \pi_{2}$ as required.

Since we have a compatible family $\left\{f_{i}\right\}_{i \in I}$ and the covering in question is canonical, 'the $f_{i}$ can be glued over $E_{1}$, i.e. there is a (unique) $g: E_{1} \rightarrow E_{2}$ such that $g \circ g_{i}=f_{i}(i \in I)$. Applying $F$, we obtain that

$$
F(g) \circ F\left(g_{i}\right)=F\left(f_{i}\right)=f \circ F\left(g_{i}\right) \quad(i \in I) .
$$

Since $\left\{F\left(S_{i}\right) \rightarrow^{F g_{i}} F\left(E_{1}\right): i \in I\right\}$ is a covering in $\mathcal{E}^{\prime}$, it follows that $F(g)=f$.

DEFINITION 2.5. Let $\delta$ be a coherent topos, $K$ a full subcategory of $\operatorname{Con}(\mathcal{E}, \mathrm{SET}),|K|$ its class of objects. Both $K$ and $|K|$ are called nice if the following conditions hold:

(i) $|K|$ is a conservative as well as a dually conservative family (see $\S 1$ );

(ii) $|K|$ is 'weakly pro-injective': whenever $M, N \in|K|, X$ is a coherent object of $\mathcal{E}, a \in M(X), b \in N(X)$ and $a \in M(A)$ imply $b \in N(A)$ for every coherent subobject $A$ of $X$, then there is a natural transformation $h: M \rightarrow N$ such that $h_{X}(a)=b$.

THEOREM 2.6. Let $\mathcal{E}$ be a coherent prime-generated topos, $K$ a nice category of models of $\mathcal{E}$. Then the evaluation functor ev: $\mathcal{E} \rightarrow(K, \mathrm{SET})$ is continuous, faithful and full.

Proof. Continuity is automatic. Faithfulness (conservativeness) is a consequence of part (i) of Definition 2.5.

Let $P$ be a coherent prime object of $\mathcal{E}, E$ an arbitrary object; we will show that ev is full on $\operatorname{Hom}_{\mathscr{E}}(P, E)$. Once we have done this, the fullness of ev will follow by 2.3 and 2.4.

Let $f$ be a morphism $\operatorname{ev}(P) \rightarrow \operatorname{ev}(E)$, i.e. a family $\left\langle f_{M}: M \in|K|\right\rangle$ of functions $f_{M}: M(P) \rightarrow M(E)$ such that

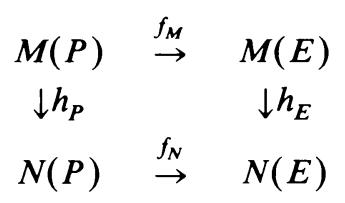

commutes whenever $h: M \rightarrow N$ is a natural transformation. Choose $M \in|K|$ and $a_{0} \in M(P)$ so that $a_{0} \notin M(A)$ for all proper subobjects $A$ of $P$; since $P$ is prime and therefore it is not covered by the family of its proper subobjects, by the conservativeness of the family $|K|$ such choices are possible (compare $\S 1$ ). Let $b_{0}=f_{M}\left(a_{0}\right) . E$ is covered by some family $\left\{Y_{i} \rightarrow^{g_{i}} E: i \in I\right\}$ of morphisms with coherent domains $Y_{i}$; $M$ preserves this covering; hence we have $i$ and some $c_{0} \in M\left(Y_{i}\right)$ such that $b_{0}=\left(M g_{i}\right)\left(c_{0}\right)$. Denote $Y_{i}$ by $Y, g_{i}$ by $g$. Let $\left\langle a_{0}, c_{0}\right\rangle$ be the element of $M(P \times Y)=M(P) \times M(Y)$ such that the canonical projections $M(P \times Y) \rightarrow M(P), M(P \times Y) \rightarrow M(Y)$ map $\left\langle a_{0}, c_{0}\right\rangle$ onto $a_{0}$ and $c_{0}$, respectively. Let $q$ be the 'type' of $\left\langle a_{0}, c_{0}\right\rangle: q$ is the set of subobjects $C$ of $P \times Y$ such that $\left\langle a_{0}, c_{0}\right\rangle \in M(C)$. 
Claim. For any $N$ in $K, a \in N(P), b \in N(E)$ we have: $f_{N}(a)=b \Leftrightarrow$ there is $c \in N(Y)$ such that $\langle a, c\rangle \in q^{N}={ }_{\mathrm{df}} \cap\{N(C): C \in q\}$ and $(N(g))(c)=b$.

Proof of ClaIM. Assume first that $f_{N}(a)=b$. By the choice of $a_{0}, a_{0} \in M(A)$ implies $a \in N(A)$ for all coherent subobjects $A$ of $P$ (simply because $a_{0} \notin M(A)$ unless $A=P$ ). Applying 2.5(ii), we have that there is $h: M \rightarrow N$ with $h_{P}\left(a_{0}\right)=a$. Define $c=h_{Y}\left(c_{0}\right)$. Consider the commutative diagram

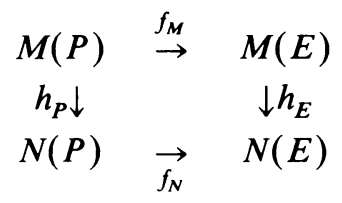

Chasing $a_{0} \in M P$ around, we get

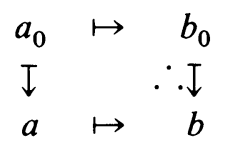

i.e. $b=h_{E}\left(b_{0}\right)$. Next, in the commutative diagram

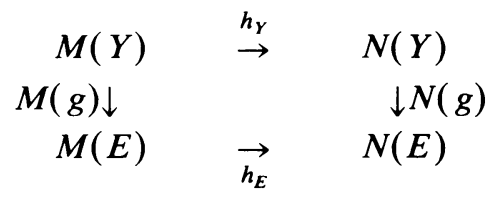

$c_{0} \in M(Y)$ chased around results in the picture

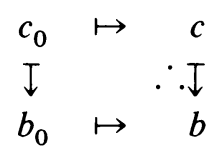

hence $b=(N(g))(c)$. Since clearly $h_{P \times Y}\left(\left\langle a_{0}, c_{0}\right\rangle\right)=\langle a, c\rangle$ and $\left\langle a_{0}, c_{0}\right\rangle \in q^{M}$ by definition, $\langle a, c\rangle \in q^{N}$ follows immediately. We have shown that the condition on the right-hand side of the equivalence holds.

Secondly, assume $\langle a, c\rangle \in q^{N}$ and $(N(g))(c)=b$. Since $q$ is the set of all coherent subobjects $C$ of $P \times Y$ with $\left\langle a_{0}, c_{0}\right\rangle \in M(C)$, we have that for all coherent subobjects $C$ of $P \times Y,\left\langle a_{0}, c_{0}\right\rangle \in M(C)$ implies $\langle a, c\rangle \in N(C)$. By 2.5(ii) again, there is $h: M \rightarrow N$ such that $h_{P \times Y}\left(\left\langle a_{0}, c_{0}\right\rangle\right)=\langle a, c\rangle$; in particular, $h_{P}\left(a_{0}\right)=a, h_{Y}\left(c_{0}\right)=c$. Looking at the second of the above two diagrams, with the new $h$ of course, we see that $h_{E}\left(b_{0}\right)=b$; finally, going to the first diagram, we conclude $f_{N}(a)=b$, proving the claim.

The following fact is a consequence of the claim: for any $N \in|K|, a \in N(P), c$, $c^{\prime} \in N(Y)$

$$
\langle a, c\rangle \in Q^{N} \text { and }\left\langle a, c^{\prime}\right\rangle \in q^{N} \quad \text { imply } \quad(N(g))(c)=(N(g))\left(c^{\prime}\right) .
$$

Indeed, for $b==_{\mathrm{df}}(N(g))(c), b^{\prime}=_{\mathrm{df}}(N(g))\left(c^{\prime}\right)$, the hypotheses imply (by the claim) that $b=f_{N}(a)=b^{\prime}$.

Now, dual conservativeness (2.5(i)) can be applied to (1), and (since $q$ is obviously closed under finite intersection) we obtain a $C \in q$ such that

$$
\langle a, c\rangle \in N(C) \text { and }\left\langle a, c^{\prime}\right\rangle \in N(C) \text { imply }(N(g))(c)=(N(g))\left(c^{\prime}\right) \text {. }
$$


[Formally, we rewrite (1) in the form $\cap_{C \in q} N(\bar{C}) \cap \cap_{C \in q} N(\overline{\bar{C}}) \subset N(X)$ for $\bar{C}=[\mathbf{C}(x, y)]_{x y y^{\prime}}, \overline{\bar{C}}=\left[\mathbf{C}\left(x, y^{\prime}\right)\right]_{x y y^{\prime}}, X=\left[g(y) \approx g\left(y^{\prime}\right)\right]_{x y y^{\prime}}$, all subobjects of $P \times$ $Y \times Y$; now, dual conservativeness gives what we want.]

Consider

$$
G \underset{\mathrm{df}}{=}[\exists y(\mathbf{C}(x, y) \wedge g(y) \approx z)]_{x z}
$$

a subobject of $P \times E$. By its definition, $G$ is interpreted in any $N \in|K|$ as follows: $\langle a, b\rangle \in N(G) \Leftrightarrow$ there is $c \in N(Y)$ such that $\langle a, c\rangle \in N(C)$ and $(N(g))(c)=b$.

We finally claim that $N(G)$ is the graph of $f_{N}$, for all $N \in K$. Since the condition $\langle a, b\rangle \in N(G)$ is obviously a weakening of that appearing in the claim, we have $f_{N}(a)=b \Rightarrow\langle a, b\rangle \in N(G)$. Assume $\langle a, b\rangle \in N(G)$; let $c \in N(Y)$ be such that $\langle a, c\rangle \in N(C)$ and $(N(g))(c)=b$. Let $b^{\prime}={ }_{\mathrm{df}} f_{N}(a)$. Then, by the claim, there is $c^{\prime} \in N(Y)$ with $\left\langle a, c^{\prime}\right\rangle \in q^{N}$ and $(N(g))\left(c^{\prime}\right)=b^{\prime}$. We have, in particular, $\left\langle a, c^{\prime}\right\rangle$ $\in N(C)$; this and $\langle a, c\rangle \in N(C)$ imply, by the choice of $C$ satisfying (2), that $(N(g))(c)=(N(g))\left(c^{\prime}\right)$, i.e. $b=b^{\prime}=f_{N}(a)$ which was to be shown.

We have shown that $G \hookrightarrow P \times E$ is functional in $N$, for every $N$ in the conservative family $|K|$; in fact $N(G)$ is the graph of $f_{N}$ for all $N \in|K|$. By 1.4, there is $g: P \rightarrow E$ in $\mathcal{E}$ such that $N(g)=f_{N}$ for all $N \in|K|$, i.e. $\operatorname{ev}(g)=f$ as desired.

COROLLARY 2.7. Every coherent prime-generated topos can be continuously and fully embedded into a functor-category $(K, \mathrm{SET})$ with a small category $K$.

Proof. Let $K_{\lambda}$ be the full subcategory of the $\lambda$-special continuous functors $\mathcal{E} \rightarrow$ SET (see $\S 1$ ) for the prime-generated coherent topos $\mathcal{E}$ and for any special cardinal $\lambda$. By 1.3 we have that $K_{\lambda}$ satisfies 2.5 (i). On the other hand, 2.5(ii) for $K_{\lambda}$ is just a reformulation of (ii) and (v) in 1.1: if the condition in 2.5(ii) holds for $a \in M(X), b \in N(X)$, then the condition in 1.1(v) will also hold for $a$ as $\vec{a}, b$ as $\vec{b}$ as seen by considering the subobjects $A={ }_{\mathrm{df}}[\varphi]_{x}, B={ }_{\mathrm{df}}[\psi]_{x}$. In other words, $K_{\lambda}$ is nice; the assertion follows by 2.6 .

LEMMA 2.8. Let $\mathcal{E}$ be a coherent topos, $\mathscr{P}=\operatorname{Coh}(\mathcal{E})$. Let $M$ and $N$ be continuous functors $\mathcal{E} \rightarrow \mathrm{SET}$, and $h$ a natural transformation $h: M \rightarrow N$. Let $M_{0}, N_{0}$ and $h_{0}$ be the restrictions of $M, N$ and $h$ to $\mathcal{P}$, i.e. the $\rho$-images of $M, N$ and $h$, respectively, with $\rho$ the restriction functor $\operatorname{Con}(\mathcal{E}, \mathrm{SET}) \rightarrow \operatorname{El}(\mathcal{P}, \mathrm{SET})$. If $h_{0}$ is an elementary embedding of $M_{0}$ into $N_{0}$, then $h$ is injective: each function $h_{E}: M(E) \rightarrow N(E)$ $(E \in|\mathcal{E}|)$ is one-to-one.

Proof. What we will actually use of $h_{0}$ being elementary is that $h$ reflects coherent subobjects: whenever $X$ is a coherent object, $A=: A \hookrightarrow{ }^{\alpha} X$ is a coherent subobject of $X$ and $\left(h_{0}\right)_{X}(x)=h_{X}(x) \in N(A)(\subset N(X))$, then $x \in M(A)(\subset$ $M(X))$. This indeed is a consequence of $h_{0}$ being elementary: $h_{0}$ (and hence $h$ ) preserves the formula $\neg \mathbf{A}(x)$, and this means exactly what we want.

Every subobject of a coherent object is the supremum of a family of coherent subobjects; this statement is contained e.g. in Lemma 6.1.3 in MR. It immediately follows that, for $h_{0}: M_{0} \rightarrow N_{0}$ elementary, $h$ reflects any subobject of a coherent object. 
Let $E$ be an arbitrary object in $\mathcal{E}$, and assume that $h_{E}(a)=h_{E}(b)$ for some $a$, $b \in M(E)$; we want to show that $a=b$. Let $\left\{X_{i} \rightarrow^{f_{i}} E: i \in I\right\}$ be a covering of $E$ by coherent objects $X_{i}(i \in I)$. Since $M$ preserves coverings, there are $i, j \in I$ and $a^{\prime} \in M\left(X_{i}\right), b^{\prime} \in M\left(X_{j}\right)$ such that $\left(M\left(f_{i}\right)\right)\left(a^{\prime}\right)=a,\left(M\left(f_{j}\right)\right)\left(b^{\prime}\right)=b$. Consider the following diagram:

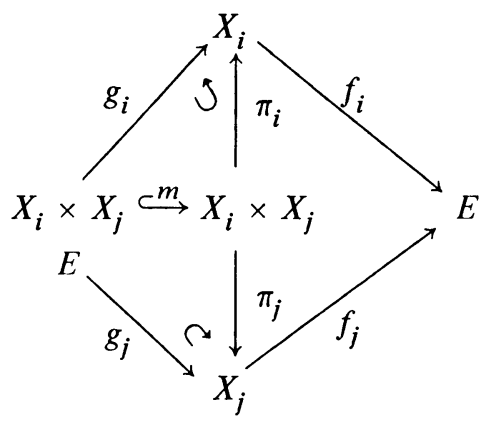

$\pi_{i}, \pi_{j}$ are canonical projections. Let $c=\left\langle a^{\prime}, b^{\prime}\right\rangle \in M\left(X_{i} \times X_{j}\right)=M\left(X_{i}\right) \times M\left(X_{j}\right)$ be such that $\left(M\left(\pi_{i}\right)\right)(c)=a^{\prime},\left(M\left(\pi_{j}\right)\right)(c)=b^{\prime}$. Putting $\bar{a}=h_{X_{i}}\left(a^{\prime}\right) \in N\left(X_{i}\right), \bar{b}=$ $h_{X_{j}}\left(b^{\prime}\right) \in N\left(X_{j}\right)$, and $\bar{c}=\langle\bar{a}, \bar{b}\rangle=h_{X_{i} \times X_{j}}(c)$, we have that $\left(N\left(f_{i}\right)\right)(\bar{a})=h_{E}(a)$, $\left(N\left(f_{j}\right)\right)(\bar{b})=h_{E}(b)$, hence $\left(N\left(f_{i}\right)\right)(\bar{a})=\left(N\left(f_{j}\right)\right)(\bar{b})$. Since $N$ preserves pullbacks, it follows that $\bar{c}$ belongs to the $N$-interpretation of the subobject $X_{i_{E}} \times X_{j} \hookrightarrow^{m} X_{i} \times X_{j}$ of the coherent object $X_{i} \times X_{j}$. Using that $h$ reflects subobjects of coherent objects, we conclude that $c \in M\left(X_{i} \times X_{j} \hookrightarrow^{m} X_{i} \times X_{j}\right) \subset M\left(X_{i} \times X_{j}\right)$. In other words, $c=$ $(M(m))(d)$ for some $d \in M\left(X_{i} \times X_{j}\right)$. Finally, $a=\left(M\left(f_{i} \circ \pi_{i} \circ m\right)\right)(d)=$ $\left(M\left(f_{i} \circ g_{i}\right)\right)(d), b=\left(M\left(f_{j} \circ \pi_{j} \circ m\right)\right)(d)=\left(M\left(f_{j} \circ g_{j}\right)\right)(d)$; hence by the commutativity of the outer rectangle in the diagram, $a=b$ as desired.

THEOREM 2.9. For any coherent prime-generated topos $\mathcal{E}$, the evaluation function ev: $\mathcal{E} \rightarrow(\mathrm{CON}(\mathcal{E}, \mathrm{SET}), \mathrm{SET})$ is continuous, conservative and full.

Proof. Only the fullness requires proof. Let $K_{\lambda}$ be as in the proof of 2.7, and consider $\mathrm{ev}_{K_{\lambda}}: \mathcal{E} \rightarrow\left(K_{\lambda}, \mathrm{SET}\right)$; for any (special cardinal) $\lambda, \mathrm{ev}_{K_{\lambda}}$ is full by the proof of 2.7. Let $K_{\lambda}^{0}$ be the full subcategory of $\operatorname{Con}(\mathcal{E}$, SET) consisting of the functors induced by the models $\operatorname{Coh}(\mathcal{E}) \rightarrow$ SET of cardinality $\leqslant \lambda$. We first show that the evaluation functor $\mathrm{ev}_{K_{\lambda}^{0}}: \mathcal{E} \rightarrow\left(K_{\lambda}^{0}, \mathrm{SET}\right)$ is full.

Let $f: \operatorname{ev}_{K_{\lambda}^{0}}\left(E_{1}\right) \rightarrow \operatorname{ev}_{K_{\lambda}^{0}}\left(E_{2}\right), f=\left\langle f_{N}: N \in\left|K_{\lambda}^{0}\right|\right\rangle$. Since $\operatorname{ev}_{K_{\lambda}}$ is full, there is $g$ : $E_{1} \rightarrow E_{2}$ such that $N(g)=f_{N}$ for all $N \in\left|K_{\lambda}\right|$. Now, by 1.1(iii) and 2.8, it follows that for any $M \in\left|K_{\lambda}^{0}\right|$, there is an injective natural transformation $h: M \rightarrow N$ into some $N \in\left|K_{\lambda}\right|$. Both of the following diagrams commute:

$$
\begin{array}{ccc}
M\left(E_{1}\right) & f_{M} & M\left(E_{2}\right) \\
h_{E_{1}} \downarrow & & \\
N\left(E_{1}\right) & & \downarrow h_{E_{2}} \\
& f_{N}=N(g) & N\left(E_{2}\right)
\end{array}
$$




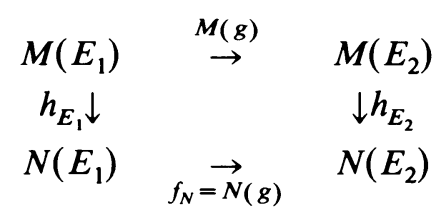

Since $h_{E_{2}}$ is $1-1$, it immediately follows that $f_{M}=M(g)$. We have shown that $f=\mathrm{ev}_{K_{\lambda}^{\mathrm{o}}}(g)$.

Finally, consider ev: $\mathcal{E} \rightarrow(K$, SET $)$ with $K=\operatorname{Con}(\mathcal{E}$, SET $)$, and let $f: \operatorname{ev}\left(E_{1}\right) \rightarrow$ $\operatorname{ev}\left(E_{2}\right), f=\left\langle f_{M}: M \in K\right\rangle$. For any special cardinal $\lambda$, there is $g_{\lambda}$ depending on $\lambda$ such that $f_{M}=M\left(g_{\lambda}\right)$ for all $M \in\left|K_{\lambda}^{0}\right|$. If $\lambda_{1}, \lambda_{2}$ are two arbitrary special cardinals, then say $\lambda_{1} \leqslant \lambda_{2}$; since $\left|K_{\lambda_{1}}^{0}\right| \subset\left|K_{\lambda_{2}}^{0}\right|, f_{M}=M\left(g_{\lambda_{1}}\right)=M\left(g_{\lambda_{2}}\right)$ for all $M \in\left|K_{\lambda_{1}}^{0}\right|$. Since $\left|K_{\lambda_{1}}^{0}\right|$ is a conservative family, it follows that $g_{\lambda_{1}}=g_{\lambda_{2}}$.

Let $g=g_{\lambda}$ for any (some) $\lambda$ as above. Since $|K|=\cup_{\lambda}\left|K_{\lambda}^{0}\right|$ (there are arbitrarily large special cardinals), it follows that $f_{M}=M(g)$ for all $M \in K ; f=\operatorname{ev}(g)$.

Let $\mathcal{R}$ be a small finitely complete regular category. $R$ is considered a site, the regular epimorphism site over $\Re$, by declaring each extremal (regular) epimorphism $A \rightarrow B$ a covering; more precisely, a family $\left\{A_{i} \rightarrow B: i \in I\right\}$ is a covering iff it contains a morphism $A_{i} \rightarrow B$ which is an extremal epi. It is shown in [2] (and can be read off from MR too) that this indeed defines a Grothendieck topology. The continuous functors $\mathscr{R} \rightarrow$ SET are exactly the exact functors.

Let $\widetilde{R}$ be the category of sheaves over this site, $\mathscr{R} \rightarrow^{e} \widetilde{\mathscr{R}}$ the canonical continuous functor. Since this topology is subcanonical, i.e. every covering is an effective epimorphic family (see SGA4 and MR), it follows that $\varepsilon$ is full and faithful. We also know that it is left exact and preserves coverings, i.e. takes an extremal epi into an extremal epi; in other words, $\varepsilon$ is exact. By its very definition, every object in the site $\mathcal{R}$ is prime. The objects $\varepsilon(A)$ in $\tilde{\mathcal{R}}$, for $A \in|\Re|$, are primes. [Any canonical covering of $\varepsilon(A)$ can be refined into a covering with domains of the form $\varepsilon\left(A_{i}\right)$, $A_{i} \in|R|$, with morphisms $\varepsilon\left(A_{i}\right) \rightarrow \varepsilon(A)$ of the form $\varepsilon\left(A_{i} \rightarrow A\right)$ (see 6.2.1 in MR); since $\varepsilon$ is also conservative (i.e. reflects coverings), it follows that one of the $A_{i} \rightarrow A$ is an extremal epimorphism, and so is $\varepsilon\left(A_{i}\right) \rightarrow \varepsilon(A)$.] Since, of course, the $\varepsilon(A)$ generate $\tilde{R}$, and also, $R$ is an algebraic site (see $\S 6.2$ in MR), $R$ is a prime generated coherent topos, and the previous results can be applied to it. A full continuous embedding of $\tilde{R}$ composed with the full, faithful and exact functor $\varepsilon$ gives a full embedding of $\mathscr{R}$ itself. This proves

COROLlARY 2.10 (BARR'S THEOREM). Any small finitely complete regular category $\Re$ can be fully and exactly embedded into a functor-category (e, SET) with a small category $\mathcal{C}$. Also, the evaluation functor $\mathrm{ev}: \mathcal{R} \rightarrow(\operatorname{Ex}(\Re, \mathrm{SET}), \mathrm{SET})$ is full, faithful and exact.

Another case when our theorem is applicable is that of coherent atomic toposes; in this case however we get more specific results: see the next section.

As we mentioned in the introduction, there are coherent toposes with continuous full embeddings into a functor category ( $C, S E T)$ that are not prime generated. On the other hand, it is not hard to see that a weakly Boolean coherent topos $\mathcal{E}$, i.e. 
one in which $\operatorname{Coh}(\mathscr{E})$ is Boolean (in $\operatorname{Coh}(\mathscr{E})$, any subobject has a Boolean complement), can have a full continuous embedding into a functor category only if it is prime-generated and, actually, atomic.

3. Atomic toposes. Atomic toposes were introduced in [3]. An object $\boldsymbol{A}$ in a topos $\mathcal{E}$ is an atom if it is nonzero and it does not have any nontrivial subobject, i.e. one that is nonzero and not the whole of $A$. $\mathscr{E}$ is atomic if the family of atoms in $\mathscr{E}$ is a family of generators for $\mathcal{E}$. Note that, obviously, every atom is prime, hence atomic toposes are prime-generated. Hence, Theorem 2.9 can be applied to coherent atomic toposes. However, in this case the situation is so much simpler that it is better to give a direct argument establishing the full continuous embedding; at the same time, we will get more specific results.

It is easy to see that the quotient of an atom is an atom; moreover that in an atomic topos, every object is a (disjoint) sum of atoms.

Proposition 3.1. (i) In a coherent atomic topos, the coherent objects are exactly the finite sums of atoms.

(ii) An atomic topos is coherent if and only if every product of finitely many atoms is a finite sum of atoms.

Proof. ad(i). Using the quasi-compactness of coherent objects and the preceding remarks, we see that every coherent object has to be a finite sum of atoms. Conversely, we claim that every atom is coherent. First of all, it is easy to see (similarly to Lemma 2.3) that the coherent atoms form a family of generators. It immediately follows that every atom is a quotient of a coherent atom. Let $A$ be a coherent atom; since $A \times A$ is coherent, it is a finite sum $\amalg_{i \in I} B_{i}$ of atoms, each of which has to be coherent. Since any subobject of $A \times A$ has to be a sum of some of the atoms $B_{i}$, it follows that every subobject of $A \times A$ is coherent. Now, let $B$ be an arbitrary atom, $A \rightarrow{ }^{f} B$ an epimorphism with $A$ a coherent atom. The kernel pair $R$ of $f$ is a coherent subobject of $A \times A$. Since the category of coherent objects is a pretopos whose inclusion in $\mathcal{E}$ is exact, it follows that $B$, as the quotient $A / R$, has to be coherent. It follows that any finite sum of atoms is coherent.

ad(ii). The 'only if' part follows from (i). The 'if' part is easily seen.

A topos is called connected if its terminal object is a atom. Since any atomic topos is a 'disjoint sum' of connected atomic toposes (as is easily seen), we can restrict attention to connected atomic toposes.

Let, until further notice, $\mathcal{E}$ be a coherent connected atomic topos, and let $M$ : $\mathcal{E} \rightarrow$ SET be a continuous functor induced by a special model $m_{0}: \mathcal{P} \rightarrow$ SET, with $\mathscr{P}=\operatorname{Coh}(\mathscr{E})$. Let $\operatorname{End}(M)$ denote the monoid of all natural transformations $M \rightarrow M(\operatorname{End}(M)$ is the full subcategory of $\operatorname{Con}(\mathcal{E}$, SET) with the single object $M)$, let $\operatorname{Aut}(M) \subset \operatorname{End}(M)$ be the group of automorphisms of $M$ and let $C$ be any submonoid of $\operatorname{End}(M)$ such that $\operatorname{Aut}(M) \subset C$. It would be easy to see that $C$ satisfies the conditions of Definition 2.5; by Theorem 2.7, we would get that ev: $\mathcal{E} \rightarrow(C, \mathrm{SET})$ is a continuous full embedding. We will obtain this result below together with a description of the essential image of $\mathscr{E}$ under ev. 
A functor $F: C \rightarrow$ SET is usually called a $C$-set for any monoid $C ;|F|$ denotes the underlying set of $F$, i.e. $F(M)$ for the unique object $M$ of $C$. In this context, we write $\gamma_{F}$ for $F(\gamma)$, with $\gamma: M \rightarrow M$ in $C$.

Given a $C$-set $F: C \rightarrow \mathrm{SET}, F$ is said to have the finite support property (f.s.p.) if the following holds: whenever $a \in|F|=F(M)$, then there are an object $X$ of $\bigodot$ and an element $x \in M(X)$ such that for any $\gamma, \delta$ in $C, \gamma_{X}(x)=\delta_{X}(x)$ implies that $\gamma_{F}(a)=\delta_{F}(a)$.

To paraphrase this condition, consider the special case $C=\operatorname{Aut}(M)$, i.e. when $C$ is a group. Let us make $C$ into a topological group by declaring that a neighborhood basis $\mathscr{B}$ of 1 , the identity element of $C$, consists of the subgroups $I(x)=\{\gamma$ $\left.\in C: \gamma_{X}(x)=x\right\}$ for any $X \in|\mathcal{E}|$ and $x \in M(X)$. It is clear that this $\mathscr{B}$ is closed under finite intersection and that $\cap \mathscr{B}=\{1\}$. Now, it is clear that in this case $F$ : $C \rightarrow$ SET has the f.s.p. just in case $F$ is a discrete $C$-set for the topological group $C$, i.e. the action $C \times|F| \rightarrow|F|:\langle\gamma, a\rangle \mapsto \gamma_{F}(a)$ of $C$ on $|F|$ is continuous with respect to the product of the topology on $C$ and of the discrete topology on $|F|$.

THEOREM 3.2. For any coherent connected atomic topos $\mathcal{E}$, for special $M: \mathcal{E} \rightarrow$ SET and any $C$ such that $\operatorname{Aut}(M) \subset C \subset \operatorname{End}(M)$, the evaluation functor ev: $\mathcal{E} \rightarrow(C, \mathrm{SET})$ is a continuous full embedding; moreover, a $C$-set $F$ is isomorphic to $\operatorname{ev}(E)$ for some $E \in|\mathcal{E}|$ if and only if $F$ has the f.s.p.

COROllary 3.3. Any coherent connected atomic topos is equivalent to the category of discrete $G$-sets for a topological group $G$.

Proof of 3.3. The corollary follows from the theorem applied to $G=\operatorname{Aut}(M)$ by the remarks preceding the theorem.

For the purposes of proving 3.2, with the notation of 3.2 we first show

LEMMA 3.4. The image under ev of every atom in $\mathcal{E}$ is an atom in (C, SET).

Proof. Let $A$ be an atom in $\mathcal{E} \cdot \operatorname{ev}(A)$ is the $C$-set such that $|\operatorname{ev}(A)|=M(A)$, and $\gamma_{\mathrm{ev}(A)}=\gamma_{A}$ for any natural transformation $\gamma: M \rightarrow M$. Assume that $F$ is a nonempty subobject of $\operatorname{ev}(A)$ (in (C, SET)); let $a \in|F| \subset M(A)$ and let $a^{\prime}$ be an arbitrary element of $M(A)$. By 3.1, we can assume without loss of generality that $A$ is an object of $\operatorname{Coh}(\mathcal{E})$. Since every atomic topos is Boolean, every formula $\varphi(x)$ (with $x$ a variable of sort $A$ ) of the language the underlying graph of $\mathcal{P}=\operatorname{Coh}(\mathcal{E}$ ) has a canonical interpretation $[\varphi]_{x} \hookrightarrow A$ such that $M_{0}\left([\varphi]_{x}\right) \subset M_{0}(A)=M(A)$ equals to $\left\{a \in M(A): M_{0} \vDash \varphi[a]\right\}$. Therefore, and by the fact that $A$ is an atom, it follows that the hypothesis of 1.1 (iv) is satisfied with $a$ and $a^{\prime}$ for $\vec{a}$ and $\vec{b}$, respectively. By 1.1(iv), there is $\gamma \in \operatorname{Aut}(M) \subset C$ such that $\gamma_{A}(a)=a^{\prime}$. Since $|F|$ is closed under the function $\gamma_{A}$, it follows that $a^{\prime} \in|F|$. Since $a^{\prime} \in M(A)$ was arbitrary it follows that $|F|=M(A)$, and the lemma is proved.

Proof of 3.2. Since 1 in $\mathcal{E}$ is an atom, it is easy to see that ev: $\mathcal{E} \rightarrow(C$, SET $)$ is conservative; it is automatically continuous. Hence ev preserves and reflects finite limits and arbitrary colimits, in particular (disjoint) sums. Since any object $X$ in $\mathcal{E}$ is a sum of atoms, $\operatorname{ev}(X)$ is a sum of subobjects each of which is the image of an atom and hence an atom itself (by 3.4). It follows that any subobject of $\operatorname{ev}(X)$ is the 
sum of some atoms and each of those atoms is the ev-image of one in $\mathcal{E}$; hence any subobject of $\operatorname{ev}(X)$ is the ev-image of a subobject of $X$. In other words, ev preserves subobject lattices: it induces an isomorphism of the subobject lattices of $X$ and $\operatorname{ev}(X)$. From this fact, the fullness of ev follows: it suffices to observe that for any $f: \operatorname{ev}(X) \rightarrow \operatorname{ev}(Y)$ the graph of $f, G \hookrightarrow \operatorname{ev}(X) \times \operatorname{ev}(Y)=\operatorname{ev}(X \times Y)$, will be the image of some $G^{\prime} \hookrightarrow X \times Y$ and since ev reflects finite limits, $G^{\prime}$ will be the graph of some morphism as well; and if $G^{\prime}$ is the graph of $f^{\prime}: X \rightarrow Y$, then clearly $\operatorname{ev}\left(f^{\prime}\right)=f$. From the same fact, it also follows that the (essential) image of $\mathcal{E}$ under ev is closed under quotients: if $\operatorname{ev}(X) \rightarrow^{p} F$ is a (regular) epimorphism in ( $C$, SET), then the kernel pair of $p$, a subobject $R \hookrightarrow \operatorname{ev}(X \times X)$, is the ev-image of some $R^{\prime} \hookrightarrow X \times X$. Since ev reflects finite limits, and $R$ is an equivalence relation on $\operatorname{ev}(X), R^{\prime}$ is an equivalence relation on $X$; if $X \rightarrow \rightarrow^{p^{\prime}} Y$ is the quotient of $R^{\prime}$ in $\mathcal{E}, \operatorname{ev}(X) \rightarrow^{\operatorname{ev}\left(p^{\prime}\right)} \operatorname{ev}(Y)$ is isomorphic to $\operatorname{ev}(X) \rightarrow^{p} F$ since both are quotients of $R$.

Turning to the 'moreover' part of 3.2, first of all it is obvious that $\operatorname{ev}(E)$ has the f.s.p. for any $E \in|\mathcal{E}|$. Conversely, let $F$ be a $C$-set with the f.s.p. Let $a \in|F|$ be arbitrary, let $X \in|\mathcal{E}|$ and $x \in M(X)$ be such that $\gamma_{X}(x)=\delta_{X}(x)$ implies $\gamma_{F}(a)=$ $\delta_{F}(a)$ for all $\gamma, \delta \in C$. Since every object in $\mathcal{E}$ is a sum of atoms, without loss of generality we can assume that $X$ is an atom. Consider the map $h: M(X) \rightarrow|F|$ defined as follows: for any $y \in M(X)$, by (the proof of) 3.4 there is $\gamma \in C$ such that $y=\gamma_{X}(x)$; define $h(y)=\gamma_{A}(a)$ with such a $\gamma$. Because of the choice of $x$ as a support for $a$, this definition does not depend on the chosen $\gamma$. It immediately follows that $h: M(X) \rightarrow|F|$ is a $C$-set map: for any $\gamma \in C$, the diagram

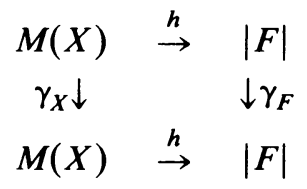

commutes. We conclude that for any $a \in|F|$ there is a morphism $h: \operatorname{ev}(X) \rightarrow F$ in $(C, \mathrm{SET})$ such that $a \in|\operatorname{Im}(h)|$. As a consequence, there is a surjective morphism $h: \amalg_{i \in I} \operatorname{ev}\left(X_{i}\right) \rightarrow F ; \amalg_{i \in I} \operatorname{ev}\left(X_{i}\right)=\operatorname{ev}\left(\amalg_{i \in I} X_{i}\right)$; and since the ev-image of $\mathcal{E}$ is closed under quotients, $F$ is isomorphic to $\operatorname{ev}(Y)$ for some $Y$ from $\mathcal{E}$.

A result related to Corollary 3.3 was obtained by André Joyal earlier than the author's findings. Joyal's theorem talks about an arbitrary connected atomic topos $\mathcal{E}$ having a point (i.e., a continuous functor $\mathcal{E} \rightarrow$ SET) and it says that $\mathcal{E}$ is equivalent to the category of discrete $G$-sets for a "localic group" $G$; this latter notion is a generalization of the notion of topological group, the same way as a complete Heyting algebra generalizes the notion of a topological space.

Theorem 3.2 was found by the author while investigating whether the category of models (continuous SET-functors) determines a coherent atomic topos; in this context, 3.2 turns out to be interesting for the case when $C$ is the full monoid $\operatorname{End}(M)$.

We now make a few remarks concerning this problem. Quite generally, it is an interesting question to what extent $\operatorname{Con}(\mathcal{E}, \mathrm{SET})$ determines the topos $\mathcal{E}$. For any theory $\mathcal{T}$, we have the classifying topos $\mathcal{E}(\mathcal{T})$ of $\mathcal{T}$ (see MR and also §5). Applied 
to $\mathscr{E}=\mathscr{E}(\mathscr{T})$, our question is a sensible way of asking to what extent the category of models determines the theory $\mathcal{T}$. It is reasonable to restrict attention to toposes with enough points; we actually consider now only coherent $\mathcal{E}$. Even with this restriction, obvious examples show that $\operatorname{Con}(\mathcal{E}, \mathrm{SET})$ may say next to nothing about $\mathscr{E}$, e.g., if $\mathscr{E}$ is the category of sheaves $\operatorname{Sh}_{p c}(\mathscr{B})$ over a Boolean algebra $\mathscr{B}$ with the precanonical (finitary) topology, then $\operatorname{Con}(\mathcal{E}$, SET) is nothing but the (discrete) set of ultrafilters on $\mathscr{B}$. If $\mathscr{B}$ and $\mathscr{B}^{\prime}$ have the same number (cardinality) of ultrafilters, $\operatorname{Con}\left(\mathrm{Sh}_{p c}(\mathscr{B}), \mathrm{SET}\right) \simeq \operatorname{Con}\left(\mathrm{Sh}_{p c}\left(\mathscr{B}^{\prime}\right), \mathrm{SET}\right)$.

To exclude this kind of triviality, we further assume that $\mathcal{E}$ is connected. Furthermore, we restrict attention to the case model theorists are mostly interested in: weakly Boolean connected coherent toposes (see the end of the last section); these are the classifying toposes of complete theories in full first order logic. Although, a negative answer to the following question (in its first, more general form) seems likely, as a matter of fact we do not have any examples to that effect.

Problem 3.5. For weakly Boolean coherent connected toposes $\mathcal{E}, \mathcal{E}^{\prime}$, does $\operatorname{Con}(\mathcal{E}, \mathrm{SET}) \simeq \operatorname{Con}\left(\mathcal{E}^{\prime}, \mathrm{SET}\right)$ imply $\mathcal{E} \simeq \mathcal{E}^{\prime}$ ? Or at least, is this true for $\mathscr{E}, \mathcal{E}^{\prime}$ both coherent connected atomic toposes?

When in the summer of 1978 the author mentioned his interest in this problem to A. Joyal, Joyal suggested the consideration of directed colimit-preserving functors $\operatorname{Con}(\mathcal{E}, \operatorname{SET}) \rightarrow$ SET. We call any functor $F: \mathcal{C} \rightarrow$ SET up-continuous if $F$ preserves directed colimits existing in $e$; Upcon( $(e, S E T)$ is the category of up-continuous functors $e \rightarrow$ SET (a full subcategory of $(C$, SET)). Now, for $C=$ $\operatorname{Con}(\mathcal{E}, \operatorname{SET})$ and $\mathrm{ev}=\mathrm{ev}_{\bigodot}: \mathcal{E} \rightarrow(\mathcal{C}, \operatorname{SET}), \operatorname{ev}(X)$ is up-continuous for any $X$ in $|\mathcal{E}|$; this fact immediately follows from the fact that directed colimits in $\operatorname{Con}(\mathcal{E}$, SET) exist and are computed as in the full functor category $(\mathcal{E}$, SET) (Tarski's union theorem, CK 3.1.13). Thus, ev: $\mathscr{E} \rightarrow(\mathcal{C}$, SET) factors through $\operatorname{Upcon}(\mathcal{C}, \mathrm{SET}) \hookrightarrow^{\text {incl. }}(\mathcal{C}, \mathrm{SET})$, and we have a functor,

$$
\mathrm{ev}^{\mathcal{\sim}}: \mathcal{E} \rightarrow \operatorname{Upcon}(\operatorname{Con}(\mathcal{E}, \mathrm{SET}), \mathrm{SET})
$$

such that ev $=$ incl. $\circ \mathrm{ev}^{\sim}$.

To indicate some minor results and some more specific problems related to up-continuous functors, we further specialize the context. In MR, §6.2, we introduced separable toposes. These coincide with the classifying toposes of theories in countable fragments of $L_{\infty}$ (for classifying toposes, see MR and also $\$ 5$ of the present paper). Separable connected coherent weakly Boolean toposes are the same as the classifying toposes of countable complete theories in full finitary logic with possibly infinitely many sorts. For brevity, we call a separable connected coherent atomic topos a special topos; special toposes are the classifying toposes of countable complete finitary theories with possibly infinitely many sorts that are $\boldsymbol{\aleph}_{0}$-categorical, i.e. for which any two models of power $\leqslant \kappa_{0}$ are isomorphic.

We note that for a coherent connected atomic topos to be separable (i.e., special) it is necessary and sufficient that there be only countably many isomorphism types of atoms in the topos.

Model theorists usually consider 1-sorted theories, or essentially equivalently, theories with finitely many sorts. A strongly special topos $\mathcal{E}$ is a special topos in 
which there are finitely many atoms $A_{1}, \ldots, A_{n}$ such that any atom in $\mathcal{E}$ is isomorphic to a subobject of a product each of whose factors is a copy of one of the $A_{i}$ 's, $i=1, \ldots, n$. Strongly special toposes are the classifying toposes of 1 -sorted countable complete $\aleph_{0}$-categorical finitary theories.

Let $\mathcal{E}$ be a special topos, $\mathscr{P}=\operatorname{Coh}(\mathcal{E})$. $\mathscr{P}$ can be taken to be a countable category (having countably many objects and morphisms), by 3.1(i) and the fact that there are only countably many nonisomorphic atoms. With such $\mathscr{P}$ in mind,

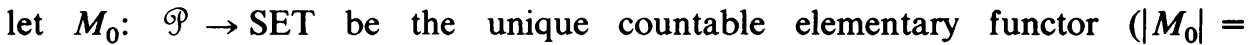
$\amalg_{X \in|\mathscr{P}|} M_{0}(X)$ is countable); in fact, it is easy to see (it is the easy part of Ryll-Nardzewski's theorem characterizing $\boldsymbol{}_{0}$-categorical theories, CK 2.3.13) that $M_{0}$ is determined up to isomorphism. Let $M \in \operatorname{Con}(\mathcal{E}$, SET $)$ be induced by $M_{0}$, and let $C=\operatorname{End}(M) . C$ has countable directed limits preserved and reflected by the inclusion $C \hookrightarrow(\mathcal{E}, \mathrm{SET})$; and it is easy to see that a functor ( $C$-set) $A$ : $C \rightarrow \mathrm{SET}$ is up-continuous just in case $A$ preserves countable directed limits; $A$ : $C \rightarrow \mathrm{SET}$ is countable if $|A|=M(A)$ is countable. Let $\operatorname{Upcon}_{\mathcal{N}_{0}}(C, \mathrm{SET})$ be the category of countable up-continuous $C$-sets; let $\mathcal{E}_{\kappa_{0}}$ be the full subcategory of $\mathcal{E}$ consisting of the objects that are countable $\left(\leqslant \kappa_{0}\right)$ sums of atoms. Using the factorization $\mathrm{ev}=$ incl. $\circ \mathrm{ev}^{\sim}$ introduced above, it is easy to see that the composite

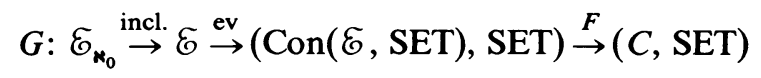

(with $F$ induced by the inclusion $C \hookrightarrow \operatorname{Con}(\mathcal{E}, \mathrm{SET})$ ) factors through $\operatorname{Upcon}_{\kappa_{0}}\left(C\right.$, SET); let $\mathrm{ev}_{\kappa_{0}}^{\sim}: \mathcal{E}_{\kappa_{0}} \rightarrow \operatorname{Upcon}_{\kappa_{0}}(C$, SET) be the functor such that $G=$ incl. ${ }^{\circ} v_{\kappa_{0}}$ for the inclusion functor incl.: $\operatorname{Upcon}_{\kappa_{0}}(C, \operatorname{SET}) \rightarrow(C, \mathrm{SET}) . \mathrm{ev}_{\boldsymbol{\kappa}_{0}}$ is simply the 'restriction' of $\mathrm{ev}^{\sim}$ introduced above to $\mathcal{E}_{\boldsymbol{N}_{0}}$ from $\mathcal{E}$ and to $C$ from $\operatorname{Con}(\mathcal{E}, \mathrm{SET})$.

Problem 3.6. Is ev $\tilde{\kappa_{0}}$, defined for a strongly special topos $\mathcal{E}$ as described, always an equivalence of categories?

By ad hoc arguments, the author has shown that for the classifying toposes of the theory of equality on an infinite set, of the theory of dense orders without endpoints and of the complete theory of any finite structure (all strongly special toposes), the answer to 3.6 is 'yes'. Subsequently, Daniel Lascar (see [8]) has found interesting general results showing that the answer is likewise 'yes' for many other strongly special toposes. In both the author's and Lascar's proofs Theorem 3.2 plays a major role. In fact, 3.2 reduces the problem of proving that $\mathrm{ev}_{\boldsymbol{\kappa}_{0}}$ is an equivalence of categories to showing that any countable up-continuous $C$-set has the f.s.p.

Despite these results, Problem 3.6 is open.

There are special toposes (that are not strongly special) for which the answer to 3.6 is 'no'; a simple example is the category of $G$-sets for the group $G=2^{\omega}$, the $\omega$-fold Cartesian power of the two-element group 2.

Clearly, if the answer to 3.6 were in general 'yes', then the answer to 3.5 restricted to strongly special toposes would also be 'yes'.

Let us call the coherent topos $\mathcal{E}$ distinguished (by its category of models) if for any coherent topos $\mathcal{E}^{\prime}$ we have that $\operatorname{Con}\left(\mathcal{E}^{\prime}, \mathrm{SET}\right) \simeq \operatorname{Con}(\mathcal{E}, \mathrm{SET})$ implies that 
$\mathcal{E}^{\prime} \simeq \mathcal{E}$. We have

Proposition 3.7. If $\mathcal{E}$ is special and $\mathrm{ev}_{\boldsymbol{\kappa}_{0}}^{-}$(associated to $\mathcal{E}$ as above) is an equivalence of categories, then $\mathcal{E}$ is distinguished.

The proof is a straightforward application of the comparison theorem, Theorem 7.1.8 in MR. In particular, 3.7 is applicable to the specific theories mentioned above and others by Lascar's results.

Finally in this section, we state

THEOREM 3.8. The conclusions of 3.2 and 3.3 are true for any separable connected atomic topos.

The proof is similar to that of 3.2 , and it is based on the well-known fact that if $M$ is a countable structure, $\vec{a}$ and $\vec{b}$ are finite tuples of elements in $M$ satisfying the same $L_{\infty \omega^{-}}$-formulas, then there is an automorphism of $M$ mapping $\vec{a}$ to $\vec{b}$.

In $\S 5$, we will describe an example of a connected atomic topos $\mathcal{E}$ without any continuous functor $\mathcal{E} \rightarrow \mathrm{SET}$; for such $\mathcal{E}$ the representation Theorem 3.2 cannot hold.

4. Barr's proof. Here we describe a proof of Barr's theorem, Corollary 2.10, which is a recasting in model-theoretic terminology of Barr's original proof. Considering the complexities of Barr's proof, we feel that our version is more easily understandable and its details are less ad hoc. From a model theoretical point of view, this proof shows the interesting fact that for so-called regular theories (see below) there always exist 'prime' models; in fact, the main construction in Barr's proof is seen to be analogous to Morley's construction of a prime model of a totally transcendental theory over an arbitrary subset of a model (see [15]). We also point out that Barr's proof establishes more than what is actually stated in [2]; the second part of 2.10 is a case in point.

Our exposition shows that our proof in $\$ 2$ is essentially different from Barr's. The latter does not seem to be adaptable to a proof of Theorem 2.9, although we lack the necessary counterexample that would conclusively show this. On the other hand, Barr's full embedding has some properties ours does not (see below).

The language of diagrams of functors $\mathcal{C} \rightarrow$ SET, used in [2], can easily be interpreted as talking about presentations of structures with similarity type the underlying graph of $\mathcal{C}$; in this way a direct relationship can be established between the category-theoretic proof in [2] and our model-theoretic argument. We will not pursue these connections; however, without such an analysis, it is far from obvious that our proof described here is 'identical' to Barr's.

We will now work with a fixed regular theory $T$ in a many-sorted language $L$ with relation and function symbols; $T$ being regular means that its axioms are all of the form

$$
\forall \vec{x}(\varphi(\vec{x}) \rightarrow \psi(\vec{x}))
$$

where $\varphi, \psi$ are primitve formulas built up from atomic formulas of $L$ using solely finite conjunction and existential quantification. For convenience, 'formula' and 'sentence' mean 'primitive formula' and 'primitive sentence' below; however, we will consider nonprimitive formulas (such as (1) above). 
The reason for considering regular theories is, of course, that the internal regular theory' $T_{\Re}^{\text {reg }}$ of a regular category $\Re$ is a regular theory; see $\S 1$.

Returning to our theory $T$, let $M$ be a model of $T, \vec{a}$ a finite tuple of elements (of various sorts) of $M$ [we imagine that the various sorts in $M$ are disjoint; otherwise we would have to carry the sorts of the elements of $M$ along as tags]. We say that $\vec{a}$ is isolated by the (primitive) $L$-formula $\varphi(\vec{x})$ if $M \vDash \varphi[\vec{a}], T \vDash \exists \vec{x} \varphi(\vec{x})$, and for any (primitive) $L$-formula $\psi(\vec{x})$ such that $M \vDash \psi[\vec{a}]$, we have that $T \vDash \forall \vec{x}(\varphi(\vec{x}) \rightarrow \psi(\vec{x}))$. Note that the notion 'isolated' depends on $T$, not just on the given model.

Theorem 4.1. Let $T$ be a regular theory. Then there is a model $M$ of $T$ such that

(i) for every model $N$ of $T$, there is a homomorphism $h: M \rightarrow N$.

In fact, more strongly we have:

(ii) every finite tuple from $M$ is isolated by some formula;

(iii) whenever $\vec{a}$ from $M$ is isolated by $\varphi(\vec{x}), N \vDash T$ and $N \vDash \varphi[\vec{b}]$, then there is a homomorphism $h: M \rightarrow N$ such that $h(\vec{a})=\vec{b}$; and

(iv) for every formula $\varphi(\vec{x})$ such that $T \vDash \exists \vec{x} \varphi(\vec{x})$, there is $\vec{a}$ in $M$ such that $\vec{a}$ is isolated by $\varphi(\vec{x})$.

Proof. Let us first note that (i) is included in the conjunction of (ii) and (iii): apply those to the empty tuple $\varnothing=\vec{a}$. By the definition, a formula isolating $\varnothing$ is a (primitive) sentence that, among others, is provable in $T$ (now the quantifiersequences $\exists \vec{x}, \forall \vec{x}$ are empty as well), so (iii) yields (i).

Let $\kappa$ be the cardinality of the set of $L$-formulas, $\left\langle c_{\alpha}: \alpha\langle\kappa\rangle\right.$ a repetition-free enumeration of new individual constants of sorts of those in $L$ such that for each sort $X$ in $L$, there are $\kappa$ distinct indices $\alpha$ with $c_{\alpha}$ being of sort $X$. Let $C=\left\{c_{\alpha}\right.$ : $\alpha<\kappa\}$. Let $\left\langle\exists x_{\alpha} \varphi_{\alpha}\left(x_{\alpha}\right): \alpha<\kappa\right\rangle$ be an enumeration of all (primitive) $L(C)$ sentences of the form $\exists x \varphi(x)$ such that, for each $\alpha, x_{\alpha}$ is of the same sort as $c_{\alpha}$ and $\exists x_{\alpha} \varphi_{\alpha}$ contains only $C$-constants $c_{\beta}$ for $\beta<\alpha$; it is easy to see that such an enumeration is possible. By induction on $\alpha<\kappa$, we define $C_{\alpha} \subset C$ and a set $\Delta_{\alpha}$ of $L\left(C_{\alpha}\right)$-sentences as follows. We put $C_{0}=\varnothing, \Delta_{0}=$ the set of primitive $L$-sentences that are consequences of $T$; for a limit $\delta<\kappa, C_{\delta}=\cup_{\alpha<\delta} C_{\alpha}, \Delta_{\delta}=\cup_{\alpha<\delta} \Delta_{\alpha}$. To define $C_{\alpha+1}, \Delta_{\alpha+1}$, we ask if $\exists x_{\alpha} \varphi_{\alpha}\left(x_{\alpha}\right)$ belongs to $\Delta_{\alpha}$. If the answer is 'no', we put $C_{\alpha+1}=C_{\alpha}, \Delta_{\alpha+1}=\Delta_{\alpha}$; if, however, the answer is 'yes', we define $C_{\alpha+1}=C_{\alpha} \cup$ $\left\{c_{\alpha}\right\}$ and $\Delta_{\alpha+1}$ to be the set of $L\left(C_{\alpha+1}\right)$-sentences $\sigma$ such that

$$
T \cup \Delta_{\alpha} \cup\left\{\varphi_{\alpha}\left(c_{\alpha}\right)\right\} \vDash \sigma .
$$

Finally, we put $C_{\kappa}=\cup_{\alpha<\kappa} C_{\alpha}, \Delta_{\kappa}=\cup_{\alpha<\kappa} \Delta_{\alpha}$. Note that every $\Delta_{\alpha}(\alpha \leqslant \kappa)$ is closed under logical consequence: if $\sigma$ is a (primitive) $L\left(C_{\alpha}\right)$-sentence and $T \cup \Delta_{\alpha} \vDash$ $\sigma$, then $\sigma \in \Delta_{\alpha}$.

As usual in a Henkin argument like this one, $\Delta_{\kappa}$ gives rise to a unique 'constant structure' $M$ whose universe consists of (equivalence classes) of constants in $C_{\kappa}$. However, we will postpone considering $M$ as long as possible since it is more convenient to talk about $\Delta_{\kappa}$ directly (which, incidentally, is the presentation of $M$ and hence is quite closely related to the diagrams of Barr). 
Let $\vec{a}$ be a finite tuple from $C_{\kappa}$. We say that $\varphi(\vec{x}) \alpha$-isolates $\vec{\alpha}$ if the following hold:

(i) $\varphi(\vec{x})$ is an $L\left(C_{\alpha}\right)$-formula,

(ii) $\varphi(\vec{a}) \in \Delta_{\kappa}$,

(iii) whenever $\psi(\vec{a}) \in \Delta_{\kappa}, \psi(\vec{x})$ is an $L\left(C_{\alpha}\right)$-formula, we have

$$
T \cup \Delta_{\alpha} \vDash \forall \vec{x}(\varphi(\vec{x}) \rightarrow \psi(\vec{x})) .
$$

Note, first of all, that if $c_{\alpha} \in C_{\kappa}$, then $c_{\alpha}$ is $\alpha$-isolated by $\varphi_{\alpha}\left(x_{\alpha}\right)$. Indeed, the first two conditions are immediate by the construction, and so is (iii) for $\psi\left(c_{\alpha}\right)$ in $\Delta_{\alpha+1}$. On the other hand, an easy inductive argument shows that for $\alpha<\beta, \Delta_{\beta}$ is a conservative extension of $\Delta_{\alpha}$, i.e. $\sigma \in \Delta_{\beta}$ implies that $\sigma \in \Delta_{\alpha}$ for any $L\left(C_{\alpha}\right)$ sentence $\sigma$; hence the facts that $\psi\left(c_{\alpha}\right) \in \Delta_{\kappa}$, and $\psi(y)$ is an $L\left(C_{\alpha}\right)$-formula imply that $\psi\left(c_{\alpha}\right) \in \Delta_{\alpha+1}$. [Although the mentioned conservativeness is almost obvious, it is a decisive fact. It can be true only because disjunctions are absent!]

LeMmA 4.2. Every finite tuple $\vec{a}$ from $C_{\kappa}$ is $\alpha$-isolated by some formula, for any $\alpha<\kappa$.

ProOF OF 4.2. Without 'oss of generality, we may assume that $\vec{a}=\left\langle c_{\gamma_{1}}, \ldots, c_{\gamma_{n}}\right\rangle$ for some $\gamma_{1}<\cdots<\gamma_{n}$. The proof will be by induction on $\gamma=\gamma_{n}$, with a fixed $\alpha$. First of all, we note that in case $\gamma<\alpha$ the formula $y_{1}=c_{\gamma_{1}} \wedge \cdots \wedge y_{n}=c_{\gamma_{n}}$ serves to $\alpha$-isolate $\vec{a}$; condition (iii) is seen to hold again because $\Delta_{\kappa}$ is conservative over $\Delta_{\alpha}$.

Assume now that $\gamma \geqslant \alpha$, let $\varphi_{\gamma}$ be the formula $\gamma$-isolating $c_{\gamma}$ (by our first remark), and let $\vec{c} \wedge \vec{d}$ be a finite repetition-free tuple of elements of $C_{\gamma}$ containing all $C$-constants from $\varphi_{\gamma}$ as well as $\vec{c}=_{\mathrm{df}}\left\langle c_{\gamma_{1}}, \ldots, c_{\gamma_{n-1}}\right\rangle$; let $\vec{y}, \vec{z}$ be tuples of variables 'matching' $\vec{c}$ and $\vec{d}$, respectively. In particular, there is a formula $\eta(\vec{y}, \vec{z}, y)$ without parameters and with at most the free variables indicated such that $\varphi_{\gamma}=\eta(\vec{c}, \vec{d}, y)$. By induction hypothesis, there is a formula $\rho(\vec{y}, \vec{z}) \alpha$-isolating $\vec{c} \wedge \vec{d}$. We claim that the formula

$$
\varphi(\vec{y}, y): \underset{\mathrm{df}}{=} \exists \vec{z}[\rho(\vec{y}, \vec{z}) \wedge \eta(\vec{y}, \vec{z}, y)]
$$

$\alpha$-isolates $\bar{a}=\vec{c}^{\wedge}\left\langle c_{\gamma}\right\rangle$. Conditions (i) and (ii) are easily seen to hold. To check (iii), let $\psi(\vec{y}, y)$ be an $L\left(C_{\alpha}\right)$-formula such that $\psi\left(\vec{c}, c_{\gamma}\right) \in \Delta_{\kappa}$. We have $T \cup \Delta_{\gamma}$ k $\forall y(\eta(\vec{c}, \vec{d}, y) \rightarrow \psi(\dot{c}, y))$ by the facts that $\alpha \leqslant \gamma$ and that $\eta(\vec{c}, \vec{d}, y) \gamma$-isolates $c_{\gamma}$. Let $\Delta$ be a finite subset of $\Delta_{\gamma}$ such that $T \cup \Delta$ deduces the same formula. By taking the conjunction of $\Delta$, and quantifying out existentially the $C$-constants other than $\vec{c}, \vec{d}$ and those in $C_{\alpha}$, we obtain an $L\left(C_{\alpha}\right)$-formula $\theta(\vec{y}, \vec{z})$ such that $\theta(\vec{c}, \vec{d}) \in \Delta_{\kappa}$ and $T \cup\{\theta(\vec{c}, \vec{d})\} \vDash \forall y(\eta(\vec{c}, \vec{d}, y) \rightarrow \psi(\vec{c}, y))$. It follows that

$$
T \vDash \forall \vec{y} y(\exists \vec{z}(\theta(\vec{y}, \vec{z}) \wedge \eta(\vec{y}, \vec{z}, y)) \rightarrow \psi(\vec{y}, y)) .
$$

Now, by $\rho(\dot{y}, \vec{z}) \alpha$-isolating $\vec{c} \wedge \vec{d}$, we have $T \cup \Delta_{\alpha} \vDash \forall \overrightarrow{y z}(\rho(\vec{y}, \vec{z}) \rightarrow \theta(\vec{y}, \vec{z}))$. This, together with (2), obviously implies

$$
T \cup \Delta_{\alpha} \vDash \forall \vec{y} y(\exists \vec{z}(\rho(\vec{y}, \vec{z}) \wedge \eta(\vec{y}, \vec{z}, y)) \rightarrow \psi(\vec{y}, y))
$$

or $T \cup \Delta_{\alpha} \vDash \forall \overrightarrow{y y}(\varphi(\vec{y}, y) \rightarrow \psi(\vec{y}, y))$ as desired. This proves the lemma. 
We return to the proof of 4.1 . Let $\vec{a}$ be any finite tuple of constants from $C_{\kappa}, \varphi(\vec{x})$ 0 -isolating $\vec{a}, N$ any model of $T$ and $\vec{b}$ elements from $N$ such that $N \vDash \varphi[\vec{b}]$. We claim that there is a map $h: C_{\kappa} \rightarrow|N|$ such that $h(\vec{a})=\vec{b}$, and for any $\vec{c}$ in $C_{\kappa}$, any $L$-formula $\psi(\vec{x}), \psi(\vec{c}) \in \Delta_{\kappa}$ implies that $N \vDash \psi[h \vec{c}]$. ( $h$ is clearly the desired homomorphism; see also later.) $h$ is constructed by transfinite recursion. Suppose that for $\beta<\alpha$ we have defined $h\left(c_{\beta}\right)$ such that we have the induction hypothesis $\psi(\vec{a}, \vec{c}) \in \Delta_{\kappa} \Rightarrow N \vDash \psi[\vec{b}, h \vec{c}]$ for any $L$-formula $\psi$ and $\vec{c}$ from $C_{\alpha}$. [Note that for $\alpha=0$, this 'induction hypothesis' is true because of the facts that $\varphi(\vec{x}) 0$-isolates $\vec{a}$ and $N \vDash T, N \vDash \varphi[\vec{b}]$.] We extend $h$ to $c_{\alpha}$ by defining $h\left(c_{\alpha}\right)$ as follows. Let $\varphi(\vec{x}, y, \vec{c})$ be a formula $\alpha$-isolating $\vec{a}^{\wedge}\left\langle c_{\alpha}\right\rangle$ with $\vec{c}$ displaying the $C_{\alpha}$-constants in the formula. Since $\exists y \varphi(\vec{a}, y, \vec{c}) \in \Delta_{\kappa}$, by the induction hypothesis $N \vDash \exists y \varphi(\vec{b}, y, h \vec{c})$; let $d=$ $h\left(c_{\alpha}\right)$ be chosen in $N$ such that

$$
N \vDash \varphi[\vec{b}, d, h \vec{c}] .
$$

Let $\psi\left(\vec{x}, y, \vec{z}^{\prime}\right)$ be any $L$-formula and $\vec{c}^{\prime}$ elements from $C_{\alpha}$ such that $\psi\left(\vec{a}, c_{\alpha}, \vec{c}^{\prime}\right) \in$ $\Delta_{\kappa}$. Since $\varphi(\vec{x}, y, \vec{c}) \alpha$-isolates $\vec{a} \wedge\left\langle c_{\alpha}\right\rangle$,

$$
T \cup \Delta_{\alpha} \vDash \forall y\left(\varphi(\vec{a}, y, \vec{c}) \rightarrow \psi\left(\vec{a}, y, \vec{c}^{\prime}\right)\right) .
$$

$N$ is a model of $T$ and, by the induction hypothesis, $N$ satisfies each formula in $\Delta_{\alpha}$ with $c_{\beta}$ interpreted as $h\left(c_{\beta}\right)$ and $\vec{a}$ interpreted as $\vec{b}$. Hence it follows that $N$ F $\forall y\left(\varphi(\vec{b}, y, h \vec{c}) \rightarrow \psi\left(\vec{b}, y, h \vec{c}^{\prime}\right)\right)$. This, together with (3), implies that

$$
N \vDash \psi\left[\vec{b}, d, h \vec{c}^{\prime}\right] .
$$

Since this was derived for an arbitrary $\psi\left(\vec{a}, c_{\alpha}, \vec{c}^{\prime}\right)$ in $\Delta_{\kappa}$ with $\vec{c}^{\prime}$ in $C_{\alpha}$, we have shown that the induction statement is true, for $\alpha+1$ in place of $\alpha$, with the chosen $d=h\left(c_{\alpha}\right)$. This completes the recursive construction of $h$.

Now, we can conclude the proof of the theorem as follows. Using the easy and familiar 'model existence' argument, we can show that there is an essentially unique $L\left(C_{\kappa}\right)$-structure $M^{*}$ such that every element in $\left|M^{*}\right|$ is of the form $c^{M^{*}}$ for some $c \in C_{\kappa}$ and such that, for any primitive $L\left(C_{\kappa}\right)$ - sentence $\sigma, M^{*} \vDash \sigma \Leftrightarrow \sigma \in \Delta_{\kappa}$. Using the fact that $T$ is regular, and that $T \cup \Delta_{\kappa} \vDash \sigma$ implies $\sigma \in \Delta_{\kappa}$ for primitive $L\left(C_{\kappa}\right)$ sentences $\sigma$, we easily deduce that $M^{*}$ is a model of $T$. Let $M$ be the $L$-reduct of $M^{*}$. Using the fact that every element of $M$ is of the form $c^{M^{*}}$ for some $c$ in $C_{\kappa}$, now it is immediate that $h$ as constructed in the last part of our proof, for given $\vec{a}$, $\varphi, N$ and $\vec{b}$, gives rise to a homomorphism as desired in part (iii) of the theorem. Similarly, part (ii) is equivalent to the lemma, with $\alpha=0$.

Finally, to see (iv), let $\varphi(\vec{x})$ be an $L$-formula such that $T \vDash \exists \vec{x} \varphi(\vec{x})$. For simplicity, we assume that $\vec{x}$ is a single variable $x$ (the argument for the general case is a straightforward variant). There is $\alpha$ such that $\exists x_{\alpha} \varphi_{\alpha}\left(x_{\alpha}\right)=\exists x \varphi(x)$. By $T \vDash \exists x \varphi(x)$, we have $\exists x \varphi(x) \in \Delta_{\alpha}$; hence, by construction $\varphi\left(c_{\alpha}\right) \in \Delta_{\alpha+1} \subset \Delta_{\kappa}$. Put $a=\left(c_{\alpha}\right)^{M^{*}}$; we claim that $a$ is isolated by $\varphi(x) . M \vDash \varphi[a]$ is clear. Suppose $M \vDash \psi[a]$; then $\psi\left(c_{\alpha}\right) \in \Delta_{\kappa}$; by the conservativeness of $\Delta_{\kappa}$ over $\Delta_{\alpha+1}, \psi\left(c_{\alpha}\right) \in \Delta_{\alpha+1}$. By definition of $\Delta_{\alpha+1}, T \cup \Delta_{\alpha} \cup\left\{\varphi\left(c_{\alpha}\right)\right\} \vDash \psi\left(c_{\alpha}\right)$, i.e. $T \cup \Delta_{\alpha} \vDash \forall x(\varphi(x) \rightarrow \psi(x))$. By taking the conjunction of a suitable finite subset of $\Delta_{\alpha}$, and quantifying out all the $C$-constants, we obtain a (primitive) $L$-sentence $\sigma$ such that $T \cup \Delta_{\alpha} \vDash \sigma$; hence $\sigma \in \Delta_{\alpha}$, and 
$T \cup\{\sigma\} \vDash \forall x(\varphi(x) \rightarrow \psi(x))$. By the conservativeness of $\Delta_{\alpha}$ over $\Delta_{0}$, we have $\sigma \in \Delta_{0}$, i.e. $T \vDash \sigma$ and hence $T \vDash \forall x(\varphi(x) \rightarrow \psi(x))$. This proves that indeed $a$ is isolated by $\varphi(x)$.

As a corollary to Theorem 4.1, we deduce what we call Barr's specific theorem (on regular categories). On the one hand, this will easily imply Barr's full exact embedding theorem (Corollary 2.10) and on the other, the specific result is not obtained by the method of special models employed in $\$ 2$.

Let $R$ be a small regular category, $M: \mathcal{R} \rightarrow$ SET an exact functor, $a \in M(X), A$ a subobject of $X$ in $R$. We say that $a$ is a generic element of $M(A)$ (or $a$ is isolated by $A$ ) if $a \in M(A) \subset M(X)$ but for no proper subobject $B$ of $A$ we have that $a \in M(B)$. The support of a subobject $A$ of $X$ is the subobject of 1 that is the image of the composite morphism $A \hookrightarrow X \rightarrow 1$.

COROLlARY 4.3 (BARR'S SPECIFIC THEOREM). Let $R$ be a small finitely complete category. There is an exact functor $M: \Re \rightarrow$ SET such that

(i) every $A \hookrightarrow X$ whose support is 1 has a generic element;

(ii) for any $X \in|R|$ and $a \in M(X)$, there is a subobject $A$ of $X$ isolating $a$;

(iii) whenever $a \in M(X)$ is a generic element of $M(A), A \hookrightarrow X, N: \Re \rightarrow$ SET is exact and $a^{\prime} \in N(A) \subset N(X)$, then there is a natural transformation $h: M \rightarrow N$ such that $h_{X}(a)=a^{\prime}$.

Proof. Let $T=T_{\mathscr{R}}^{\mathrm{reg}}$ be the internal regular theory of $\mathscr{R}$ (see $\S 1$ ). Applying 4.1 to $T$, we obtain $M$ as desired; indeed (i) is a consequence of 4.1(iv) (applied to $\mathbf{A}(x)$ as $\varphi(\vec{x})$ ) and (ii) and (iii) are identical to (ii) and (iii), respectively, in 4.1 (by passing from a primitive formula $\varphi(x)$ to its canonical interpretation $\left.[\varphi]_{x} \hookrightarrow X\right)$.

SeCOND PROOF OF BARR's THEOREM, COROLlary 2.10. Let $R$ be as in 4.3. For $P$ an arbitrary subobject of 1 , consider the comma-category $R / P$ (see e.g. Mac Lane [9]) and apply 4.3 to $R / P$; we obtain (as is easily seen) an exact $M_{P}: R \rightarrow$ SET satisfying (ii) and (iii) in 4.3 , and (i) $)_{P}$ which is (i) in 4.2 with 1 replaced by $P$. (Alternatively, one can apply 4.1 to the theory whose axioms are those of $T_{\Re}$ plus $P$, to get the same result.) We now prove that for any full subcategory $K$ of $\operatorname{Ex}(\Re, \mathrm{SET})$ such that $M_{P} \in|K|$ for all $P \hookrightarrow 1$, we have that $\mathrm{ev}=\mathrm{ev}_{K}: \mathcal{R} \rightarrow$ $(K, \mathrm{SET})$ is exact, conservative (hence faithful) and full. Exactness is automatic; conservativeness follows easily from (i) ${ }_{P}$ for $M_{P}$. To show fullness, let $f=$ $\left\langle f_{M}\right\rangle_{M \in|K|}$ be a morphism: $\operatorname{ev}(A) \rightarrow \operatorname{ev}(B)(A, B \in|\Re|)$; in other words, we have that the diagram

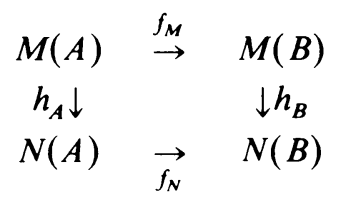

is commutative for all $h: M \rightarrow N$.

Let $P$ be the support of $A$ (as a subobject of itself), let $M=M_{P}$, and (by (i) $)_{P}$ let $a \in M(A)$ be a generic element of $M(A)$. Let $b=f_{M}(a)$, and let $c=\langle a, b\rangle \in$ $M(A) \times M(B)=M(A \times B)$ be the element whose images under the canonical 
projections $M(A \times B) \rightarrow^{M\left(\pi_{A}\right)} M(A), M(A \times B) \rightarrow^{M\left(\pi_{B}\right)} M(B)$ are $a$ and $b$, respectively. Let $G \hookrightarrow A \times B$ be a subobject of $A \times B$ such that $c$ is a generic element of $M(G)$. We claim that for all $N \in|K|$, the graph of $f_{N}$, $\operatorname{graph}\left(f_{N}\right) \subset N(A \times B)$, coincides with $N(G) \subset N(A \times B)$. To prove the claim, first suppose $c^{\prime}=\left\langle a^{\prime}, b^{\prime}\right\rangle$ $\in \operatorname{graph}\left(f_{N}\right)$, i.e. $b^{\prime}=f_{N}\left(a^{\prime}\right)$. By (iii) in 4.3, since $a$ is generic in $M(A)$, there is $h$ : $M \rightarrow N$ such that $h_{A}(a)=a^{\prime}$. By the above commutative diagram, it follows that $b^{\prime}=h_{B}(b)$, hence $h_{A \times B}(c)=c^{\prime}$. Since $c \in M(G)$, it follows that $c^{\prime} \in N(G)$ as desired. Conversely, suppose $c^{\prime}=\left\langle a^{\prime}, b^{\prime}\right\rangle \in N(G)$. Since $c$ is generic in $M(G)$, by (iii) in 4.2 there is $h: M \rightarrow N$ such that $h_{A \times B}(c)=c^{\prime}$; hence $h_{A}(a)=a^{\prime}$ and $h_{B}(b)=b^{\prime}$. Applying again the commutative diagram, we obtain that $f_{N}\left(a^{\prime}\right)=b^{\prime}$, i.e. $c^{\prime} \in \operatorname{graph}\left(f_{N}\right)$ as desired. This completes the proof of the claim.

Now, it easily follows (similarly as in the proof of 2.6), that $G$ is the graph of a morphism $g: A \rightarrow B$ in $\Re$ and that $f_{N}=N(g)$ for $N \in|K|$, i.e. $f=\operatorname{ev}(g)$. This completes the proof of 2.10 .

A few more remarks about Barr's theorem and Barr's proof. Let $\Re$ be as above. First of all, Barr's specific theorem can be used to prove the result that, for $\tilde{\mathcal{R}}$ the category of regular epimorphism sheaves over $\mathscr{R}, \tilde{\Re}$ has full continuous embeddings into functor categories $(K, \mathrm{SET})$; in fact, the lifting of the evaluation functor ev: $\mathscr{R} \rightarrow(K, \mathrm{SET}), \mathrm{ev}^{\sim}: \tilde{\mathscr{R}} \rightarrow(K, \mathrm{SET})$ works whenever $K$ is such as in the preceding proof. The proof is just slightly more complicated than the preceding proof, and it is similar to the proof of 2.7. Of course, this result is a strengthening of Barr's Theorem 2.10 (and a special case of 2.9 at the same time).

Secondly, taking $|K|$ to be the set $\left\{M_{P}: P\right.$ a subobject of 1$\}$ in the preceding proof, one sees that $R$ has a full exact embedding into a functor category $(K$, SET) with a category $K$ whose objects are indexed by the subobjects of 1 ; in particular, if 1 has only itself as subobjects, then $K$ is a monoid. Applying this to a small abelian category $\mathcal{Q}$, one immediately obtains (see Barr [2]) that $\mathcal{Q}$ has a full exact embedding into the category of (left) $R$-modules for a ring $R$ : the Mitchell full embedding theorem. We note that our proof in $\$ 2$ can also be made to yield these results.

Thirdly, (as Barr also mentions in [2]) the embedding ev: $\mathcal{R} \rightarrow(K, \mathrm{SET})$ of the preceding proof is such that the image of every object $X$ or $R, \operatorname{ev}(X)$, is a (regular) quotient of a representable functor: for $P$ the support of $X, M=M_{P}$ and $x$ a generic element of $M(X)$, we have the natural transformation $\nu: \operatorname{Hom}_{K}(M,-) \rightarrow$ ev(X) defined by $\nu_{N}(h)=h_{X}(x)$ for any $h: M \rightarrow N$; by property (iii) in 4.3 of $M=M_{P}, \nu$ is surjective. We could have derived a similar conclusion from our arguments in $\$ 2$ as well.

Finally, one peculiar property of Barr's embedding: if we take $|K|=\left\{M_{P}: P\right.$ a subobject of 1$\}$ as in the second remark, then the embedding ev: $R \rightarrow(K$, SET $)$ will preserve all the existing (infinite) intersections of subobjects in $R$; this is an easy consequence of the fact that in $M_{P}$, every element is 'isolated', i.e. $M_{P}$ has the property (ii) in 4.3 . This last property is definitely not shared by the embedding constructed in $\$ 2$. In fact, it is more or less contradictory to the property of dual conservativeness in 2.5 (although note that $K$ does have the other properties in 2.5). This last property was also noted by Barr [2]. 
5. Classifying toposes and the Malitz-Gregory example of an atomic topos without points. In the preceding sections, model theory was always used in applications to given categories; however, logic can also be used to construct toposes with various desired properties. The main goal of this section is to describe an example of a connected atomic topos without points, i.e. without any continuous functor to SET; the topos will be the so-called classifying topos of an infinitary theory described by J. Malitz [14] and J. Gregory [5]. First, we state some generalities in outline; the details are explained in MR.

In MR, the notion of the classifying topos $\mathcal{E}(T)$ of a theory $T$ is explained for the case when $T$ is an infinitary coherent theory, i.e. its axioms are of the form $\forall \vec{x}(\varphi(\vec{x}) \rightarrow \psi(\vec{x}))$ where $\vec{x}$ is a finite sequence of variables, $\varphi$ and $\psi$ are infinitary coherent, or $L_{\infty \omega}^{g}$-formulas built up from atomic formulas by finitary conjunction, infinitary disjunction and existential quantification. The classifying topos $\mathscr{E}(T)$ is defined essentially by a universal property: $\mathscr{E}(T)$ is equipped with a 'canonical' $\mathcal{E}(T)$-valued model $M: T \rightarrow \mathcal{E}(T)$ of $T$ such that for any topos $\mathcal{E}$ and any $\mathcal{E}$-valued model $N: T \rightarrow \mathcal{E}$ there is an essentially unique (up to a unique isomorphism) continuous functor $F: \mathcal{E}(T) \rightarrow \mathcal{E}$ such that

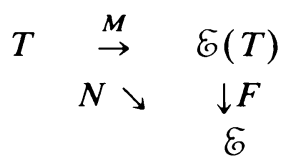

is commutative. The existence of $\mathcal{E}(T)$ is, in itself, almost immediate on general grounds; however, $\mathcal{E}(T)$ also has a concrete syntactical description based on the formulas of the theory; this is given in Chapter 8 of MR.

We extend the notion of a classifying topos to more general theories as follows. Let $\mathcal{F}$ be a fragment of $L_{\infty \omega}$, i.e. a set of $L_{\infty \omega}$-formulas containing all atomic formulas of $L$ and closed under subformulas and substitution (see p. 71 in MR). Here we will also assume that all fragments are closed under finite conjunction and existential quantification. A theory is a pair $(T, \mathcal{F})$ with $\mathscr{F}$ a fragment (of $L_{\infty}$ ) and $T$ a set of axioms of the form $\forall \vec{x}(\varphi(\vec{x}) \rightarrow \psi(\vec{x}))$ with $\varphi$ and $\psi$ formulas of $\mathscr{F}$. By a Boolean $\mathcal{E}$-model of $T$ we mean an interpretation of the underlying language $L$ of $T$ in $\mathcal{E}$ that satisfies each axiom of $T$ under the Boolean interpretation, i.e. when negation $(\neg)$ is interpreted as Boolean complement, and $\wedge, \rightarrow$ and $\forall$ are interpreted as their Boolean equivalents expressed by $\neg, \bigvee$ and $\exists$ as in classical logic. Note that for an arbitrary topos $\mathcal{E}$ and an arbitrary interpretation $M: L \rightarrow \mathcal{E}$ of $L$ in $\mathcal{E}$, a formula $\varphi$ of $\mathcal{F}$ need not even be interpretable in the Boolean interpretation; it is just in case the requisite Boolean complements corresponding to the particular negated subformulas of $\varphi$ exist in $\mathcal{E}$. On the other hand, $\varphi$ might very well be interpretable in $M$, and $M$ might even satisfy $T$, without $\mathcal{E}$ being Boolean, i.e. without all subobjects in $\mathcal{E}$ having Boolean complements.

Now, the classifying topos $\mathcal{E}(T, \mathscr{F})$ of the theory $(T, \mathscr{F})$ has a similar definition as before: it comes equipped with a Boolean $\mathcal{E}(T, \mathscr{F})$-model $M: T \rightarrow \mathcal{E}(T, \mathscr{F})$ such that for any Boolean $\mathcal{E}$-model $N: T \rightarrow \mathcal{E}$, there is an essentially unique continuous $F: \mathcal{E}(T, \mathscr{F}) \rightarrow \mathcal{E}$ such that $F \circ M=N$. 
The existence, and description, of $\mathscr{E}(T, \mathscr{F})$ for a general theory $(T, \mathscr{F})$ can be inferred easily from the infinitary coherent case; in fact, the seemingly more general notion is not essentially more general. The reason for this is the obvious but important fact that any elementary functor $M: \Re \rightarrow \mathscr{F}$ automatically preserves all existing Boolean complements in $\Re$ : if $A$ is a subobject of $X$ in $\Re, B$ is its Boolean complement, then $A \wedge B=0=\bigvee \varnothing=$ the empty subobject of $X$; and $A \vee B=$ $X=\wedge \varnothing$ the maximal subobject of $X ; M$, being elementary, preserves these relationships; hence $M(B)$ is the Boolean complement of $M(A)$ in $\mathscr{F}$. Given a theory $(T, \mathscr{F})$, we produce an infinitary coherent theory $T^{*}$ in a larger language: first, we rewrite every formula to use only $\exists, \wedge, \vee$ and $\neg$ as usual; second, to eliminate $\neg$, we introduce a new predicate $R_{\neg \varphi}(\vec{x})$ for each formula $\neg \varphi(\vec{x})$ in $\mathscr{F}$; third, we replace any 'outermost' subformula of the form $\neg \varphi(\vec{x})$ in a formula $\sigma$ by $R_{\neg \varphi}(\vec{x})$, with the resulting formula being denoted by $\sigma^{*}$, and finally, as the axioms of $T^{*}$ we have the *-transforms of the axioms of $T$ as well as all axioms of the form

$$
\left.\forall \vec{x} \text { (true } \rightarrow \varphi^{*}(\vec{x}) \vee R_{\neg \varphi}(\vec{x})\right), \quad \forall \vec{x}\left(\varphi^{*}(\vec{x}) \wedge R_{\neg \varphi}(\vec{x}) \rightarrow\right. \text { false) }
$$

with $\neg \varphi(\vec{x})$ in $\mathscr{F}$. It is easy to see that $\mathscr{E}\left(T^{*}\right)$ qualifies as $\mathscr{E}(T, \mathscr{F})$.

To illustrate the effect of this definition, we mention that the classifying toposes of theories in full finitary first order logic (as $\mathscr{F}$ ) are exactly those coherent toposes in which the coherent objects form a Boolean category. In addition, the terminal object in the topos is an atom (the topos is connected) just in case the theory is complete.

Next we turn to theories whose classifying topos is connected atomic.

For a set $T$ of axioms in $L_{\infty \omega \omega}$, and a sentence $\sigma$ of $L_{\infty \omega \omega}, T \vdash \sigma$ means that $\sigma$ is (synactically) deducible from $T$ (see e.g. Chapter 5 in MR); by the Boolean completeness theorem for $L_{\infty \omega}$ (see e.g. loc. cit.), this is equivalent to saying that every Boolean-valued model of $T$ is a model of $\sigma$. A theory $(T, \mathscr{F})$ is called complete if it is consistent, i.e. $T \nvdash$ false and if for any sentence $\sigma$ of $\mathscr{F}$, either $T \vdash \sigma$ or $T \vdash \neg \sigma$. Given a theory $\mathscr{T}=(T, \mathscr{F})$, a formula $\varphi(\vec{x}) \in \mathscr{F}$ is called complete (in variables $\vec{x}$ ) with respect to $\mathscr{T}$ if $T \vdash \exists \vec{x} \varphi(\vec{x})$ and for all $\psi(\vec{x})$ in $\mathscr{F}$ (with free variables $\vec{x}$ ) either $T \vdash \forall \vec{x}(\varphi(\vec{x}) \rightarrow \psi(\vec{x}))$ or $T \vdash \forall \vec{x}(\varphi(\vec{x}) \rightarrow \neg \psi(\vec{x})$ ) (it is not assumed that the displayed sentences belong to $\mathscr{F})$. The theory $(T, \mathscr{F})$ is called atomic if for every finite sequence $\vec{x}$ of variables (of various sorts),

$$
T \vdash \forall \vec{x} \bigvee\{\varphi(\vec{x}): \varphi(\vec{x}) \text { is a complete formula in } \vec{x}\}
$$

(again, it is not assumed that the displayed sentence belongs to $\mathscr{F}$ ).

Proposition 5.1. For any theory $(T, \mathscr{F})$, the classifying topos $\mathcal{E}(T, \mathscr{F})$ is connected atomic if and only if $(T, \mathscr{F})$ is complete atomic.

Proof. For the proof, we will have to use some facts about the construction of the classifying topos. With $M_{0}: T \rightarrow \mathcal{E}(T, \mathscr{F})$ the canonical model of $T$, we write $[\varphi]_{\vec{x}}$ for $\left(M_{0}\right)_{\vec{x}}(\varphi)$ (see Chapter 2 in MR); for any $\varphi$ in $\mathscr{F}$, the canonical interpretation $[\varphi]_{\vec{x}}$ is Boolean (i.e., all negations etc. in $\varphi$ are evaluated in $\mathcal{E}(T, \mathscr{F})$ in the Boolean sense). $[\varphi]_{\vec{x}}$ is a subobject of an appropriate product object in $\mathcal{E}(T, \mathscr{F})$; depending on the context, we may mean the domain of this subobject when talking 
about $[\varphi]_{\vec{x}}$. One fact is that $[\varphi]_{\vec{x}} \leqslant[\psi]_{\vec{x}}$ (as subobjects) just in case

$$
T \vdash \forall \vec{x}(\varphi(\vec{x}) \rightarrow \psi(\vec{x})) .
$$

Another is that the topos $\mathcal{E}(T, \mathscr{F})$ is generated by (the objects of the form) $[\varphi]_{\vec{x}}, \varphi$ in $\mathscr{F}$. Finally, every subobject of an object $E$ of the form $M\left(X_{1}\right) \times \cdots \times M\left(X_{n}\right)$ (the $X_{i}$ sorts of $L, n \geqslant 0$ ) (call such distinguished objects) is the supremum (disjunction) of subobjects of $E$ of the form $[\varphi]_{\vec{x}}, \varphi$ in $\mathscr{F}, \vec{x}=\left\langle x_{1}, \ldots, x_{n}\right\rangle, x_{i}$ of sort $X_{i}$. All these facts are in Chapter 8 of MR; the last fact also uses the assumption that $\mathscr{F}$ is closed under finite conjunction and existential quantification.

Assume $(\mathcal{F}, T)$ is complete atomic. By the third fact applied to $1=$ the empty product, and also the first fact, it follows that $\mathcal{E}(\mathscr{F}, T)$ is connected (note that completeness includes consistency implying that $1 \neq 0$ ). One similarly infers that for a complete formula $\varphi(\vec{x}),[\varphi(\vec{x})]_{\vec{x}}$ is an atom. The atomicity condition on the theory implies that every distinguished object, and in fact, every subobject of a distinguished object, is covered by a family of atoms. Finally, by the second fact above, it follows that the family of atoms is a family of generators for $\mathcal{E}(T, \mathscr{F})$ as required.

Conversely, assume that $\mathcal{E}(T, \widetilde{F})$ is connected atomic. By the third fact, it easily follows that each distinguished object must be the supremum of subobjects of the form $[\varphi]_{\vec{x}}$ which are atoms at the same time. But if $[\varphi]_{\vec{x}}$ is an atom, then $\varphi(\vec{x})$ is a complete formula, as is seen by the first fact. This shows that $(T, \mathscr{F})$ is atomic; completeness is clear.

We note that in case of a complete atomic theory $(T, \mathcal{F})$, the classifying topos $\mathcal{E}(T, \mathscr{F})$ does not essentially depend on the fragment $\mathscr{F}:$ if $\mathscr{F}^{\prime}$ is another fragment over the same language as $\mathscr{F}, \mathscr{F} \subset \mathscr{F}^{\prime}$, then $\mathcal{E}\left(T, \mathscr{F}^{\prime}\right)$ is the same as $\mathcal{E}(T, \mathscr{F})$ with the same canonical model $M: T \rightarrow \mathcal{E}(T, \mathscr{F})$. This is immediate by the defining universal property of $\mathcal{E}\left(T, \mathcal{F}^{\prime}\right)$ and the fact that $\mathscr{E}(T, \mathcal{F})$, as any atomic topos, is Boolean. We note that this is not necessarily true for general theories $(T, \mathscr{F})$.

Let $(T, \mathscr{F})$ be an arbitrary theory. Using the defining universal property of $\mathcal{E}(T, \mathscr{F})$, we see that the continuous functors $\mathcal{E}(T, \mathscr{F}) \rightarrow$ SET are essentially in one-one correspondence with the ordinary (SET-) models of the theory $(T, \mathcal{F})$. Hence, by Proposition 5.1, in order to construct a connected atomic topos $\mathcal{E}$ without any continuous functor $\mathcal{E} \rightarrow \mathrm{SET}$, it is (necessary and) sufficient to construct a complete atomic theory $(T, \mathscr{F})$ without any model.

Such a theory is described in Gregory [5]; Gregory's example is a minor variant of one in Malitz [14] (but Gregory uses the example for different purposes). Unfortunately, both authors fall short of stating (and proving) that the theory is atomic, although Malitz proves a fact from which it is possible to infer (by a forcing argument, similar to the one used by Gregory) the atomicity of the theory. In the final analysis, there is no saving by relying on the forcing argument, since the basic elementary argument going into Malitz's proof is the same as the one needed for the direct proof of the atomicity of the theory. Since there has been considerable interest in the existence of a 'pointless' atomic topos, we will describe the essential points of the necessary arguments.

A full binary tree is any structure $(T,<)$ isomorphic to $\left(2^{<\omega}, \subset\right)$, the finite 0 -1-sequences partially ordered by the 'initial segment of' relation. A branch of 
such $\langle T,\langle\rangle$ is a maximal linearly ordered (by $<$ ) subset of $T$. Let $I$ be an arbitrary set; let $L_{I}$ be the following two-sorted language: the two sorts are denoted by $T$ ('tree') and $B$ ('branch'); there are binary relations < " $\subset T \times T$ ", $\varepsilon$ " $\subset T \times B$ " (i.e., the first argument of $\varepsilon$ is of sort $T$, the second of sort $B$ ) and for each $\alpha \in I$, a unary relation $Q_{\alpha}$ " $\subset B$ " (Gregory uses $A$ for $B$ and $R$ for $\varepsilon$ ). The set $\Phi_{I}$ of axioms is described informally as follows:

(i) $(T,<)$ is a full binary tree;

(ii) distinct elements $b$ of $B$ correspond to distinct branches $\{t \in T: t \in b\}$ of $T$;

(iii) the sets $Q_{\alpha}$ are pairwise disjoint;

(iv) the union for all $\alpha \in I$ of the sets $Q_{\alpha}$ is $B$;

(iv) $)_{\alpha}^{\prime}$ each $Q_{\alpha}$ is dense in the sense that for any $t$ in $T$ there is $b \in Q_{\alpha}$ such that $t \in b$ (in particular, each $Q_{\alpha}$ is nonempty).

For the (straightforward) exact formulation of the axioms, see [5, pp. 448-449]. With a large enough fragment $\mathscr{F}_{I}$, consider the theory $\mathscr{T}_{I}=\left(\Phi_{I}, \mathscr{F}_{I}\right)$. The example we want is $\widetilde{T}_{I}$ with a set $I$ of cardinality greater than the continuum, $2^{\aleph_{0}}$.

It is immediate that $\mathcal{T}_{I}$ (for such $I$ ) cannot have a model: in any model, the denotation of $(T,<)$ is necessarily a full binary tree; hence there are $2^{k_{0}}$ branches of $(T,<)$, and there cannot be more than $2^{\alpha_{0}}$ many pairwise disjoint nonempty sets of branches.

On the other hand, if $I$ is say countably infinite then it is easy to construct a model of $\mathscr{T}_{I}$ (see also [14]). 'Imitating' this construction, for an arbitrary $I$ one can construct a $\mathscr{B}$-valued model of $\mathscr{T}_{I}$ where $\mathscr{B}$ is the Boolean algebra of the regular open sets of the product space $I^{\omega}$, with $I$ equipped with the discrete topology; it follows that $\sigma_{I}$ is consistent, i.e. $\sigma_{I} \not t$ false. [Alternatively, one can pass to the Boolean valued universe $\mathbf{V}^{\mathscr{B}}$, with $\mathscr{B}$ as described; in $\mathbf{V}^{\mathscr{B}}, I$ is countable, hence in $\mathbf{V}^{\mathscr{B}}, \mathcal{T}_{I}$ has a model; by the absoluteness of consistency, it follows that $\mathcal{T}_{I}$ is consistent (in V).]

In the remainder of this section, we deal with the atomicity of $\mathscr{T}_{I}$. We first develop a general criterion for atomicity which is of independent interest.

Let $\mathscr{T}=(T, \mathscr{F})$ be a theory. An atomic system for $\mathscr{T}$ consists of sets $A_{\vec{x}}$ of formulas $\varphi(\vec{x})$ in $\mathscr{F}$, one set for each finite tuple $\vec{x}$ of distinct variables (of arbitrary sorts), such that the following conditions hold:

(v) $T \vdash \forall \vec{x} \bigvee_{\varphi \in A_{\vec{x}}} \varphi(\vec{x})$;

(vi) for any two distinct members $\varphi, \varphi^{\prime}$ of $A_{\vec{x}}, T \vdash \forall \vec{x}\left(\varphi(\vec{x}) \wedge \varphi^{\prime}(\vec{x}) \rightarrow\right.$ false);

(vii) whenever $\varphi(\vec{x} x)$ is in $A_{\vec{x} x}$, there is a $\bar{\varphi}(\vec{x})$ in $A_{\vec{x}}$ such that

$$
T \vdash \forall \vec{x}(\bar{\varphi}(\vec{x}) \leftrightarrow \exists x \varphi(\vec{x}, x)) ;
$$

(viii) when $\vec{x}=\varnothing$ is the empty tuple, $A_{\varnothing}$ is the singleton $\{$ true $\}$;

(ix) each $\varphi(\vec{x}) \in A_{\vec{x}}$ is complete with respect to atomic formulas: for every atomic formula $\theta(\vec{x})$ (in at most the free variables $\vec{x}$ ), we have $T \vdash \forall \vec{x}(\varphi(\vec{x}) \rightarrow \theta(\vec{x})$ ), or $T \vdash \forall \vec{x}(\varphi(\vec{x}) \rightarrow \neg \theta(\vec{x}))$.

PROPOSITION 5.2. $(T, \mathscr{F})$ is complete atomic if and only if it is consistent and has an atomic system.

Proof. It is easy to see that the set of complete formulas in a given tuple $\vec{x}$ of free variables qualifies for the $A_{\vec{x}}$, in case $(T, \mathscr{F})$ is atomic. 
Suppose $(T, \mathscr{F})$ is consistent and has the atomic system $\left\langle A_{\vec{x}}: \vec{x} \ldots\right\rangle$. We prove that every $\varphi(\vec{x}) \in A_{\vec{x}}$ is a complete formula, except maybe for the 'consistency condition' $T \vdash \exists \vec{x} \varphi(\vec{x})$. Once we have shown this, it follows by (viii) that $(T, \mathscr{F})$ is complete. Then for the subset $A^{\prime}{ }_{\vec{x}}$ of $A_{\vec{x}}$ consisting of those $\varphi \in A_{\vec{x}}$ such that $T \vdash \exists \vec{x} \varphi(\vec{x})$, we have by (v) that $T \vdash \forall x \bigvee\left\{\varphi(\vec{x}): \varphi \in A_{\vec{x}}^{\prime}\right\}$ (because for $\varphi \in A_{\vec{x}}-$ $\left.A_{\vec{x}}^{\prime}, T \vdash \forall \vec{x}(\neg \varphi(\vec{x}))\right)$, and also that every $\varphi \in A_{\vec{x}}^{\prime}$ is complete, showing that $(T, \mathcal{F})$ is complete atomic.

It remains to do the following: by induction on the complexity of the arbitrary formula $\theta(\vec{x})$, we prove that every $\varphi(\vec{x}) \in A_{\vec{x}}$ decides $\theta$ : either $T \vdash \forall \vec{x}(\varphi(\vec{x}) \rightarrow \theta(\vec{x}))$ or $T \vdash \forall \vec{x}(\varphi(\vec{x}) \rightarrow \neg \theta(\vec{x}))$. For atomic formulas, this is assumption (ix). We have to consider three inductive cases, corresponding to $\neg, \bigvee$ and $\exists$, because the other logical operators are expressible in terms of these. The statement for $\neg \theta(\vec{x})$ is a trivial consequence of that for $\theta(\vec{x})$. Let $\theta(\vec{x})=\bigvee_{i \in I} \theta_{i}(\vec{x})$; by the induction hypothesis, if $\varphi(\vec{x}) \in A_{\vec{x}}$, then either $T \vdash \forall \vec{x}\left(\varphi \rightarrow \theta_{i}\right)$ for some $i \in I$, in which case $T \vdash \forall \vec{x}(\varphi \rightarrow \theta)$, or for all $i \in I, T \vdash \forall \vec{x}\left(\varphi \rightarrow \neg \theta_{i}\right)$ in which case

$$
T \vdash \forall \vec{x}\left(\varphi \rightarrow \bigwedge_{i \in I} \neg \theta_{i}\right),
$$

i.e. $T \vdash \forall \vec{x}(\varphi \rightarrow \neg \theta)$. Each step we have made in this argument, and also each similar one below, is obviously justified for " $T \vdash(\cdot)$ " meant as the Boolean-valued semantical consequence relationship.

Before turning to the last case, we show the following:

$$
T \vdash \forall \vec{x}\left(\varphi(\vec{x}) \rightarrow \forall x \bigvee\left\{\eta(\vec{x} x): \eta \in A_{\vec{x} x}^{\varphi}\right\}\right)
$$

where $\varphi(\vec{x}) \in A_{\vec{x}}$, and $A_{\vec{x} x}^{\varphi}=\left\{\eta \in A_{\vec{x} x}: \bar{\eta}=\varphi\right\}$ (for $\bar{\eta}$, see condition (vii)). Namely, if $\eta \in A_{\vec{x} x}-A_{\vec{x} x}^{\varphi}$, then $\bar{\eta}$, being different from $\varphi$, is contradictory to $\varphi$ (see (vi)); hence it follows that $T \vdash \forall \vec{x} x(\varphi(\vec{x}) \rightarrow \neg \eta(\vec{x} x))$. This fact, together with (v), now easily implies our claim.

Let, finally, $\theta(\vec{x})$ be $\exists x \psi(\vec{x} x)$, and $\varphi(\vec{x}) \in A_{\vec{x}}$. If there is $\eta \in A_{\vec{x} x}^{\varphi}$ such that $T \vdash \forall \vec{x} x(\eta \rightarrow \psi)$, then $T \vdash \forall \vec{x}(\exists x \eta \rightarrow \exists x \psi)$ and hence by $T \vdash \forall \vec{x}(\varphi \leftrightarrow \exists x \eta)$ (see (vi)), $T \vdash \forall \vec{x}(\varphi \rightarrow \exists x \psi)$ as desired. If, on the other hand, there is no such $\eta$, then by the induction hypothesis, $T \vdash \forall \vec{x} x(\eta \rightarrow \neg \psi)$ for all $\eta \in A_{\vec{x} x}^{\varphi}$; by (1) now it follows that $T \vdash \forall \vec{x}(\varphi(\vec{x}) \rightarrow \forall x \neg \psi)$, i.e. $T \vdash \forall \vec{x}(\varphi(\vec{x}) \rightarrow \neg \exists x \psi(\vec{x} x))$ as desired.

The point of the last proposition is, of course, that it gives an explicit set of deducibilities one has to check for atomicity, whereas in the definition of a complete formula, there is a deducibility to check for all formulas of the fragment. Incidentally, it also shows the 'independence on the fragment' of the atomicity of a theory noted earlier.

Returning to the Malitz-Gregory theory $\mathcal{T}_{I}$, we are left with the task of exhibiting an atomic system for $\mathcal{T}_{I}$.

Let $L_{n}(u)(n<\omega)$ be a formula expressing that $u$ (in $T$ ) is on level $n$ in the tree (the predecessors of $u$ form a set of order-type $n)$. Let $L_{n}\left(u, u^{\prime}\right)(n<\omega)$ express that $u$ and $u^{\prime}$ (in $T$ ) 'meet on level $n$ ', i.e. that the set of those $t$ such that $t<u$ and $t<u^{\prime}$ has order type $n$. There are two other versions of $L_{n}\left(u, u^{\prime}\right): L_{n}^{\prime}\left(v, v^{\prime}\right)$ and $L_{n}^{\prime \prime}(u, v)$ for 'branches' $v$ and $v^{\prime}$, and with tree-element $u$ and branch $v$; e.g. 
$L_{n}^{\prime \prime}(u, v)$ expresses that the set of $t \in T$ such that $t<u$ and $t \in v$ has order type $n$. For simplicity, we omit the primes, and talk about $L_{n}(u, v)$, etc.

Let $\left(T_{0},<\right)$ be a concrete (the) full binary tree, $\vec{c}=\left\langle c_{0}, \ldots, c_{m-1}\right\rangle$ a finite tuple of elements $t \in T_{0}$ and branches $b \in B\left(T_{0}\right)$ of $T_{0}$. The pattern of $\vec{c}$ consists of specifying (i) the level of each $t$ in $\vec{c}$ and (ii) the level where any two $c_{i}$ meet; this is always well defined unless the two $c_{i}$ 's are identical branches. More precisely, the pattern of $\vec{c}$ is the set of formulas

$$
\begin{aligned}
& \left\{L_{n}\left(x_{i}\right): c_{i} \in T \text { is on level } n, i<m, n<\omega\right\} \\
& \quad \cup\left\{L_{n}\left(x_{i}, x_{j}\right): c_{i}, c_{j} \text { meet on level } n, i, j<m, i \neq j, n<\omega\right\} \\
& \quad \cup\left\{x_{i}=x_{j}: c_{i} \in B\left(T_{0}\right), c_{j} \in B\left(T_{0}\right), c_{i}=c_{j}, i, j<m, i \neq j\right\} .
\end{aligned}
$$

LEMMA 5.3 ('BACK-AND-FORTH PROPERTY'). Suppose $\vec{c}$ has the same pattern as $\vec{d}=\left\langle d_{0}, \ldots, d_{m-1}\right\rangle$ in $\left(T_{0},<\right)$ and $d \in T_{0} \cup B\left(T_{0}\right)$ is arbitrary. Let $Q \subset B\left(T_{0}\right)$ be an arbitrary dense set of branches (see (iv) $)_{\alpha}^{\prime}$. Then there is $c$ such that $\vec{c}^{\wedge}\langle c\rangle$ and $\vec{d} \wedge\langle d\rangle$ have the same pattern, and in addition, in case $d$ is a branch distinct from each $d_{i}, c \in Q$.

Proof. Let $\vec{c}=\left\langle c_{0}, \ldots, c_{m-1}\right\rangle, \vec{d}=\left\langle d_{0}, \ldots, d_{m-1}\right\rangle$. If $d=d_{i}$ for some $i$, put $c={ }_{\mathrm{df}} c_{i}$; this choice clearly will do. Assume $d \neq d_{i}$ for all $i<m$. Then for each $i, d$ meets $d_{i}$ at a unique finite level $n_{i}$; if $d \in T_{0}$ and $n$ is the level of $d$, then $n_{i} \leqslant n$. Let $i_{0}<m$ be such that $n_{i_{0}}$ is maximal among the $n_{i}, i<m$. Let $c$ be chosen such that the level of $c$ is the same as the level of $d$, if they are both in $T_{0}$, and $c$ is a branch in $Q$ if $d$ is a branch; moreover such that $c$ meets $c_{i_{0}}$ on level $n_{i_{0}}$. Such a choice is possible: just branch off $c_{i_{0}}$ at level $n_{i_{0}}$ in the other of the two directions available at that level and continue arbitrarily up to the desired level; if $d$ is a branch, by the density of $Q$, we can choose $c$ in $Q . c$ cannot meet any $c_{i}$ on a level strictly higher than $n_{i_{0}}$, and $c$ cannot be identical to any $c_{i}$ : if it did with some $c_{i}, c_{i}$ would meet $c_{i_{0}}$ on level $n_{i_{0}}$; hence $d_{i}$ would meet $d_{i_{0}}$ on level $n_{i_{0}}$, and since there are only two branchings at level $n_{i_{0}}$ on $d_{i_{0}}$, $d$ would have met $d_{i}$ on a level higher than $n_{i_{0}}$. Hence, $c$ meets any $c_{i}$ on the same level as $c_{i_{0}}$ meets $c_{i}$, hence as $d_{i_{0}}$ meets $d_{i}$, hence as $d$ meets $d_{i}$.

A pattern $p(\vec{x})$ in the variables $\vec{x}=\left\langle x_{0}, \ldots, x_{m-1}\right\rangle$ (of both sorts of $\mathscr{T}_{I}$ ) is the pattern of some $\vec{c}$ in $\left(T_{0},<\right)$. A labeled pattern $\varphi(\vec{x})$ in $\vec{x}$ is the conjunction of a set of the form $p(\vec{x}) \cup\left\{Q_{\alpha_{i}}\left(x_{i}\right): i<m, x_{i}\right.$ of sort $\left.B\right\}$ such that $p(\vec{x})$ is a pattern, $\alpha_{i} \in I$ for each $i<m$, and also, if $x_{i}=x_{j}$ belongs to $p(\vec{x})$, then $\alpha_{i}=\alpha_{j}$.

Let $A_{\vec{x}}$ be the set of labeled patterns in the variables $\vec{x}$. We claim that $\left\langle A_{\vec{x}}\right.$ : $\vec{x} \ldots\rangle$ is an atomic system for $\mathscr{T}_{I}$. The verification is completely elementary; we consider only the most interesting part, condition (vii).

Given a labeled pattern $\varphi(\vec{x}, x)=\bigwedge \Sigma$ we define $\bar{\varphi}(\vec{x})$ as $\bigwedge \bar{\Sigma}$ where $\bar{\Sigma}$ is $\Sigma$ minus all formulas mentioning $x$. In particular, let the pattern $p(\vec{x}, x)$ involved in $\Sigma$ be that of $\vec{d} \wedge\langle d\rangle$ in $\left(T_{0},<\right)$; then that involved in $\bar{\Sigma}$ is the pattern $p(\vec{x})$ of $\vec{d}$. It is clear that $\Phi_{I} \vdash \forall \vec{x}(\exists x \varphi(\vec{x}, x) \rightarrow \bar{\varphi}(\vec{x}))$; it remains to show $\Phi_{I} \vdash \forall \vec{x}(\bar{\varphi}(\vec{x}) \rightarrow$ $\exists x \varphi(\vec{x}, x))$. 
Let $I_{0}$ be the finite set of $\alpha \in I$ mentioned in $\varphi(\vec{x}, x)$. Let $\Phi^{\prime}$ be the set of axioms (i)-(iii) plus (iv) $)_{\alpha}^{\prime}$ for $\alpha \in I_{0} . \Phi^{\prime}$ is a countable theory in $L_{\omega_{1} \omega}$ (has only countable conjunctions and disjunctions). We will actually show the stronger statement $\Phi^{\prime} \vdash \forall \vec{x}(\bar{\varphi}(\vec{x}) \rightarrow \exists x \varphi(\vec{x}, x))$; by the completeness theorem for $L_{\omega_{1} \omega}$ (see e.g. MR, 5.1.7), it is enough to show that the displayed sentence is true in any (ordinary) model of $\Phi^{\prime}$. But any model of $\Phi^{\prime}$ is isomorphic to one of the following kind: $\left(T_{0},<\right)$ as the interpretation and $T$ and $<$, a set of branches of $T_{0}$ as the interpretation of $B$, dense disjoint subsets $Q_{\alpha}$ of $B$ for $\alpha \in I_{0}$, and ordinary membership as $\varepsilon$. To show that $\forall \vec{x}(\varphi(\vec{x}) \rightarrow \exists x \varphi(\vec{x}, x))$ is true in this model, let $\vec{c}$ be arbitrary satisfying $\varphi(\vec{x})$. Let $Q_{\alpha_{m}}(x)$ be the $Q_{\alpha}$ formula for the variable $x$ mentioned in $\varphi$, i.e. $Q_{\alpha_{m}}(x) \in \Sigma$. Hence, with the $\vec{d} \wedge d$ introduced above, $\vec{c}$ and $\vec{d}$ have the same pattern. By 5.3, let $c$ be such that $\vec{c}^{\wedge}\langle c\rangle$ and $\vec{d}^{\wedge}\langle d\rangle$ have the same pattern, and in addition, $c \in Q_{\alpha_{m}}$ in case $d$ is a branch, $d$ distinct from each $d_{i}$. If $d$ happens to be equal to some branch $d_{i}$, then $c$ equals $c_{i}$; now $\alpha_{m}=\alpha_{i}$ (see the definition of labeled patterns), hence automatically $c \in Q_{\alpha_{m}}$. Clearly, we have shown that $\vec{c}^{\wedge} c$ satisfies the labeled pattern $\varphi(\vec{x}, x)$ as desired.

This completes our verification of the atomicity of the Malitz-Gregory theory.

In summary, we have shown:

THEOREM 5.4. There is a connected atomic topos without points.

\section{REFERENCES}

SGA4 1. M. Artin, A. Grothendieck and J. L. Verdier, Théorie des topos et cohomologie etale des schémas, Lecture Notes in Math., vols. 269, 270, Springer-Verlag, Berlin and New York, 1972.

2. M. Barr, Exact categories, Exact Categories and Categories of Sheaves, (M. Barr, P. A. Grillet and D. H. Van Osdol), Lecture Notes in Math., vol. 236, Springer-Verlag, Berlin and New York, 1971, pp. 1-120.

3. M. Barr and R. Diaconescu, Atomic toposes, J. Pure Appl. Algebra 17 (1980), 1-24.

CK 4. C. C. Chang and H. J. Keisler, Model theory, North-Holland, Amsterdam, 1973.

5. J. Gregory, Incompleteness of a formal system for infinitary finite-quantifier formulas, J. Symbolic Logic 36 (1971), 445-455.

6. P. Johnstone, Topos theory, Academic Press, New York, 1977.

7. H. J. Keisler, Model theory of infinitary logic, North-Holland, Amsterdam, 1971.

8. D. Lascar, On the category of models of a complete theory, J. Symbolic Logic (to appear).

9. S. Mac Lane, Categories for the working mathematician, Springer-Verlag, Berlin and New York, 1971.

MR 10. M. Makkai and G. E. Reyes, First order categorical logic, Lecture Notes in Math., vol. 611, Springer-Verlag, Berlin and New York, 1977.

11. M. Makkai, On full embeddings. I, J. Pure Appl. Algebra 16 (1980), 183-195.

12. __ Full continuous embeddings of Grothendieck toposes, Notices Amer. Math. Soc. 26 (1979), 79T-A113.

13. __ The topos of types, Logic Year 1979-80, The University of Connecticut, Lecture Notes in Math., vol. 859, Springer-Verlag, Berlin and New York, 1981, pp. 157-201.

14. J. Malitz, The Hanf number for complete $L_{\omega, \omega}$ sentences, The Syntax and Semantics of Infinitary Languages, Lecture Notes in Math., vol. 72, Springer-Verlag, Berlin and New York, 1968, pp. 166-181.

15. M. D. Morley, Categoricity in power, Trans. Amer. Math. Soc. 114 (1965), 514-538.

Department of Mathematics, McGill University, Montreal, Quebec, Canada H3A 2K6 